\title{
Palladium-Catalyzed ipso-Borylation of Aryl Sulfides with Diborons
}

M. Bhanuchandra, Alexandre Baralle, Shinya Otsuka, Keisuke Nogi, Hideki Yorimitsu,* and Atsuhiro Osuka

Department of Chemistry, Graduate School of Science, Kyoto University

Sakyo-ku, Kyoto 606-8502, Japan

yori@kuchem.kyoto-u.ac.jp

\section{Table of Contents}

Instrumentation and Chemicals

Optimization of Borylation Conditions

Experimental Procedures and Characterization Data

References

${ }^{1} \mathrm{H}$ and ${ }^{13} \mathrm{C}$ NMR Spectra

S11-41

\section{Instrumentation and Chemicals}

${ }^{1} \mathrm{H}$ NMR $(600 \mathrm{MHz})$ and ${ }^{13} \mathrm{C}$ NMR $(151 \mathrm{MHz})$ spectra were taken on a JEOL ECA-600 spectrometer. Chemical shifts $(\delta)$ are reported in parts per million, relative to residual chloroform at $7.26 \mathrm{ppm}$ for ${ }^{1} \mathrm{H}$ and relative to $\mathrm{CDCl}_{3}$ at $77.0 \mathrm{ppm}$ for ${ }^{13} \mathrm{C}$ in $\mathrm{CDCl}_{3}$. Carbons bearing boron atoms are not observed due to the quadrupolar relaxation mechanism of the ${ }^{11} \mathrm{~B}$ nucleus and consequently are not reported here. Mass spectra were determined on a Bruker micrOTOF II-KR (ESI) spectrometer. TLC analyses were performed on commercial glass plates bearing a $0.25-\mathrm{mm}$ layer of Merck silica gel $60 \mathrm{~F}_{254}$ or boric-acid-coated TLC plates (BTLC plates). ${ }^{1}$ UV lamp (254 nm), iodine, or phosphomolybdic acid (PMA) were used for staining the TLC plates. B-silica gel ${ }^{1}$ or silica gel [Kanto, $60 \mathrm{~N}$ (neutral, 63-210 $\mu \mathrm{m}$ )] were used for column chromatography.

Unless otherwise noted, materials obtained from commercial suppliers were used without further purification. Aryl sulfides were purchased from Sigma-Aldrich, TCI, or Wako Pure Chemical Industries, Ltd. Bis(pinacolato)diboron was purchased from Wako Pure Chemical Industries, Ltd. Pd-PEPPSI-IPr and lithium hexamethyldisilazide [ $\left.\mathrm{LiN}\left(\mathrm{SiMe}_{3}\right)_{2}\right]$ were purchased from Sigma-Aldrich. Dehydrated THF was purchased from Wako Pure Chemical Industries, Ltd. and stored under nitrogen. 
Table S1. Optimization of Borylation Conditions ${ }^{\text {a }}$<smiles>Cc1ccc(S(C)(C)C)cc1</smiles>

$1 a$

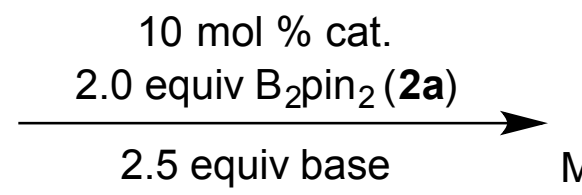

solvent, $80{ }^{\circ} \mathrm{C}, 15 \mathrm{~h}$<smiles>Cc1ccc(Br)cc1</smiles>

$3 a$

\begin{tabular}{|c|c|c|c|c|}
\hline entry & catalyst & base & solvent & yield $/ \%^{b}$ \\
\hline 1 & Pd-PEPPSI-IPr & $\mathrm{LiN}\left(\mathrm{SiMe}_{3}\right)_{2}$ & THF & $0^{\mathrm{c}}$ \\
\hline 2 & Pd-PEPPSI-IPr & $\mathrm{LiN}\left(\mathrm{SiMe}_{3}\right)_{2}$ & $\mathrm{THF}$ & $23^{\mathrm{d}}$ \\
\hline 3 & Pd-PEPPSI-IPent & $\mathrm{LiN}\left(\mathrm{SiMe}_{3}\right)_{2}$ & THF & 64 \\
\hline 4 & SingaCycle-A2 & $\mathrm{LiN}\left(\mathrm{SiMe}_{3}\right)_{2}$ & THF & 38 \\
\hline 5 & $\mathrm{PdCl}_{2}(\mathrm{dppf})$ & $\mathrm{LiN}\left(\mathrm{SiMe}_{3}\right)_{2}$ & $\mathrm{THF}$ & 0 \\
\hline 6 & Sphos-Pd-G2 & $\mathrm{LiN}\left(\mathrm{SiMe}_{3}\right)_{2}$ & THF & 0 \\
\hline 7 & Ruphos-Pd-G2 & $\mathrm{LiN}\left(\mathrm{SiMe}_{3}\right)_{2}$ & THF & 0 \\
\hline 8 & Pd-PEPPSI-IPr & none & THF & 0 \\
\hline 9 & Pd-PEPPSI-IPr & LiTMP & THF & 49 \\
\hline 10 & Pd-PEPPSI-IPr & $\mathrm{LiCl}$ & THF & 0 \\
\hline 11 & Pd-PEPPSI-IPr & LiOEt & $\mathrm{THF}$ & 25 \\
\hline 12 & Pd-PEPPSI-IPr & $\mathrm{LiN}\left(\mathrm{SiMe}_{3}\right)_{2}$ & $\mathrm{CH}_{3} \mathrm{CN}$ & 0 \\
\hline 13 & Pd-PEPPSI-IPr & $\mathrm{LiN}\left(\mathrm{SiMe}_{3}\right)_{2}$ & $\mathrm{DMF}$ & 0 \\
\hline 14 & Pd-PEPPSI-IPr & $\mathrm{LiN}\left(\mathrm{SiMe}_{3}\right)_{2}$ & DMSO & 0 \\
\hline 15 & Pd-PEPPSI-IPr & $\mathrm{LiN}\left(\mathrm{SiMe}_{3}\right)_{2}$ & Diglyme & 15 \\
\hline 16 & Pd-PEPPSI-IPr & $\mathrm{LiN}\left(\mathrm{SiMe}_{3}\right)_{2}$ & $m$-dimethoxybenzene & 41 \\
\hline
\end{tabular}




\section{Experimental Procedure and Characterization Data:}

\section{Preparation of B-TLC: ${ }^{1}$}

B-TLC plates were prepared from commercially available aluminium sheets bearing a 0.25 $\mathrm{mm}$ layer of Merck Silica gel $60 \mathrm{~F}_{254}$ through immersion in $5 \% \mathrm{w} / \mathrm{v} \mathrm{B}(\mathrm{OH})_{3} /$ ethanol for 20 min and subsequent desiccation in vacuo for $1 \mathrm{~h}$ at ambient temperature.

Preparation of B-silica gel (with slight modification from the reported procedure): ${ }^{1}$

Silica gel [150 g, Kanto, $60 \mathrm{~N}$ (neutral, 63-210 $\mu \mathrm{m}$ )] was immersed in ethanol containing 5\% w/v boric acid $(27.5 \mathrm{~g}$ in $550 \mathrm{~mL})$ for $1 \mathrm{~h}$ with gentle shaking. After removal of solvent by filtration, the gel was washed with ethanol $(600 \mathrm{~mL})$ and hexane $(600 \mathrm{~mL})$ dried in vacuo over $1.5 \mathrm{~h}$ at $60{ }^{\circ} \mathrm{C}$ and used for column chromatography.

\section{Synthesis of Aryl Sulfides: ${ }^{2}$}

To a solution of $\mathrm{NaOH}(7.5 \mathrm{mmol})$ in dry ethanol $(6.0 \mathrm{~mL})$ was added arenethiol $(5.0 \mathrm{mmol})$ and the reaction mixture was stirred for $30 \mathrm{~min}$. Alkyl iodide $(7.5 \mathrm{mmol})$ was added dropwise and the resulting solution was stirred for $3 \mathrm{~h}$ at room temperature. The reaction mixture was quenched with ice-cold water $(3 \mathrm{~mL})$ and extracted with dichloromethane $(3 \times$ $20 \mathrm{~mL}$ ). The organic layer was separated, dried over $\mathrm{Na}_{2} \mathrm{SO}_{4}$, and concentrated under vacuum. The crude residue was purified by using column chromatography on silica gel (Hexane/EtOAc) to provide aryl alkyl sulfide.

Compounds 1a-d ${ }^{3} 1 \mathbf{1},{ }^{4} 1 \mathbf{k}-\mathbf{l},{ }^{5} 1 \mathbf{1 m},{ }^{3} 1 \mathbf{1 n},{ }^{6} 1 \mathbf{1 o}-\mathbf{q},{ }^{3} 1 \mathbf{r},{ }^{7} 1 \mathbf{s},{ }^{3}$ and $\mathbf{1 t}{ }^{12}$ are known compounds and showed the identical spectra according to the literature.

\section{Synthesis of 9-(4-methylsulfanylphenyl)-9H-carbazole (1e): 8}

To a solution of dibenzo[b,d]thiophene 5,5-dioxide (216 $\mathrm{mg}, 1.0 \mathrm{mmol})$ and 4methylsulfanylaniline $(278 \mathrm{mg}, 2.0 \mathrm{mmol})$ in dioxane $(3 \mathrm{~mL})$ was added dropwise a solution of KHMDS (0.5 M in toluene, $6.0 \mathrm{~mL}, 3.0 \mathrm{mmol})$ under argon atmosphere at an ambient temperature. The resulting solution was stirred at $80{ }^{\circ} \mathrm{C}$ for $16 \mathrm{~h}$. The reaction mixture was quenched with sat. $\mathrm{NH}_{4} \mathrm{Cl}$ solution $(5 \mathrm{~mL})$ and extracted with ethyl acetate $(3 \times 20 \mathrm{~mL})$. The organic layer was separated, dried over $\mathrm{Na}_{2} \mathrm{SO}_{4}$, and concentrated under vacuum. The crude residue was purified using column chromatography on silica gel $($ Hexane/EtOAc $=20 / 1)$ to provide 9-(4-methylsulfanylphenyl)-9H-carbazole (1e, $192 \mathrm{mg}, 0.66 \mathrm{mmol}, 66 \%$ yield). 


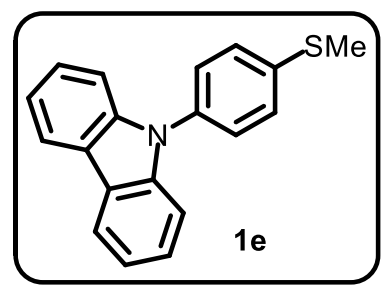

\section{9-(4-Methylsulfanylphenyl)-9H-carbazole (1e):}

Pale yellow solid. $\mathrm{mp}=152-153{ }^{\circ} \mathrm{C} ;{ }^{1} \mathrm{H}$ NMR $\left(\mathrm{CDCl}_{3}\right) \delta 8.15(\mathrm{~d}, J=8.4 \mathrm{~Hz}, 2 \mathrm{H}), 7.51-7.46$ $(\mathrm{m}, 4 \mathrm{H}), 7.43-7.37(\mathrm{~m}, 4 \mathrm{H}), 7.29(\mathrm{dt}, J=7.2,1.8 \mathrm{~Hz}, 2 \mathrm{H}), 2.59$ (s, 3H) ppm; ${ }^{13} \mathrm{C} \mathrm{NMR}$ $\left(\mathrm{CDCl}_{3}\right) \delta 140.9,137.9,134.7,127.7,127.6,125.9,123.3,120.3,119.9,109.7,15.9$ ppm; HRMS (APCI-MS, Positive) $(\mathrm{m} / z)$ : [M] ${ }^{+}$Calcd for $\mathrm{C}_{19} \mathrm{H}_{15} \mathrm{NS}, 289.0925$; found, 289.0922.

\section{Synthesis of 4-benzyloxyphenyl methyl sulfide (1g): ${ }^{9}$}

4-Hydroxythioanisole (701 mg, $5.0 \mathrm{mmol})$, benzyl bromide $(1.03 \mathrm{~g}, 6.0 \mathrm{mmol}), \mathrm{K}_{2} \mathrm{CO}_{3}(1.38$ $\mathrm{g}, 10.0 \mathrm{mmol})$, and $\mathrm{KI}(124 \mathrm{mg}, 0.75 \mathrm{mmol})$ were taken in an oven-dried Schlenk flask under an argon atmosphere. Acetone $(10 \mathrm{~mL})$ was added to this mixture. The resulting solution was refluxed for $12 \mathrm{~h}$. The reaction mixture was diluted with water and extracted with ethyl acetate. The organic layer was separated, dried over $\mathrm{Na}_{2} \mathrm{SO}_{4}$, and concentrated under vacuum. The crude residue was purified using column chromatography on silica gel (Hexane/EtOAc $=$ 19/1) to provide 4-benzyloxyphenyl methyl sulfide (1g, $723 \mathrm{mg}, 3.14 \mathrm{mmol}$, 63\% yield).

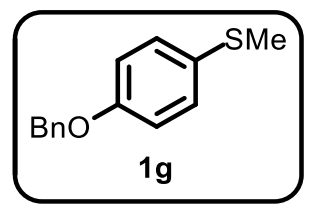

\section{4-Benzyloxyphenyl methyl sulfide (1g):}

Colorless solid. $\mathrm{mp}=77-78{ }^{\circ} \mathrm{C} ;{ }^{1} \mathrm{H} \mathrm{NMR}\left(\mathrm{CDCl}_{3}\right) \delta 7.43(\mathrm{~d}, J=7.8 \mathrm{~Hz}, 2 \mathrm{H}), 7.40(\mathrm{t}, J=7.8$ $\mathrm{Hz}, 2 \mathrm{H}), 7.34(\mathrm{t}, J=7.8 \mathrm{~Hz}, 1 \mathrm{H}), 7.28(\mathrm{~d}, J=9.0 \mathrm{~Hz}, 2 \mathrm{H}), 6.93(\mathrm{~d}, J=9.0 \mathrm{~Hz}, 2 \mathrm{H}), 5.06$ (s, 2H), 2.45 (s, 3H) ppm; ${ }^{13} \mathrm{C} \mathrm{NMR}\left(\mathrm{CDCl}_{3}\right) \delta 157.3,136.8,130.0,129.1,128.6,128.0,127.4$, 115.5, 70.1, 17.9 ppm; HRMS (APCI-MS, Positive) $(\mathrm{m} / \mathrm{z})$ : [M] $]^{+}$Calcd for $\mathrm{C}_{14} \mathrm{H}_{14} \mathrm{OS}$, 230.0765; found, 230.0767.

\section{Synthesis of 4-(methoxymethoxy methyl sulfide (1h): ${ }^{9}$}


To a solution of 4-hydroxythioanisole $(701 \mathrm{mg}, 5.0 \mathrm{mmol})$ and $\mathrm{NaH}(180 \mathrm{mg}, 7.50 \mathrm{mmol})$ in DMF $(8 \mathrm{~mL})$ at $0{ }^{\circ} \mathrm{C}$ was added chloromethyl methyl ether $(805 \mathrm{mg}, 10.0 \mathrm{mmol})$ dropwise under argon atmosphere. The resulting solution was stirred at room temperature for $12 \mathrm{~h}$. The reaction mixture was quenched with ice-cold water $(3 \mathrm{~mL})$ and extracted with ethyl acetate $(3$ $\times 20 \mathrm{~mL}$ ). The organic layer was separated, dried over $\mathrm{Na}_{2} \mathrm{SO}_{4}$, and concentrated under vacuum. The crude residue was purified using column chromatography on silica gel $($ Hexane/EtOAc $=6 / 1)$ to provide 4-(methoxymethoxy)phenyl methyl sulfide $(\mathbf{1 h}, 890 \mathrm{mg}$, $4.85 \mathrm{mmol}, 97 \%$ yield).

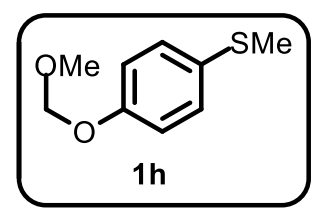

\section{4-(Methoxymethoxy)phenyl methyl sulfide (1h):}

Colorless liquid; ${ }^{1} \mathrm{H}$ NMR $\left(\mathrm{CDCl}_{3}\right) \delta 7.25(\mathrm{~d}, J=9.0 \mathrm{~Hz}, 2 \mathrm{H}), 6.99(\mathrm{~d}, J=9.0 \mathrm{~Hz}, 2 \mathrm{H}), 5.15$ $(\mathrm{s}, 2 \mathrm{H}), 3.47$ (s, 3H), 2.45 (s, 3H) ppm; ${ }^{13} \mathrm{C} \mathrm{NMR}\left(\mathrm{CDCl}_{3}\right) \delta 155.6,130.3,129.7,117.0,94.5$, 55.9, 17.7 ppm; HRMS (APCI-MS, Positive) (m/z): [M] $]^{+}$Calcd for $\mathrm{C}_{9} \mathrm{H}_{12} \mathrm{O}_{2} \mathrm{~S}, 184.0558$; found, 184.0561 .

\section{Synthesis of triisopropyl(4-methylsulfanylphenoxy)silane (1i): ${ }^{9}$}

To a solution of 4-hydroxythioanisole $(701 \mathrm{mg}, 5.0 \mathrm{mmol})$ and imidazole $(1.02 \mathrm{~g}, 15.0 \mathrm{mmol})$ in DCM $(8 \mathrm{~mL})$ at $0{ }^{\circ} \mathrm{C}$ was added triisopropylsilyl chloride $(1.93 \mathrm{~g}, 10.0 \mathrm{mmol})$ under argon atmosphere. The resulting solution was stirred at room temperature for $12 \mathrm{~h}$. The reaction mixture was quenched with ice-cold water $(3 \mathrm{~mL})$ and extracted with ethyl acetate $(3 \times 20$ $\mathrm{mL}$ ). The organic layer was separated, dried over $\mathrm{Na}_{2} \mathrm{SO}_{4}$, and concentrated under vacuum. The crude residue was purified using column chromatography on silica gel (Hexane/EtOAc $=$ 19/1) to provide triisopropyl(4-methylsulfanylphenoxy)silane (1i, $1.36 \mathrm{~g}, 4.61 \mathrm{mmol}, 92 \%$ yield).

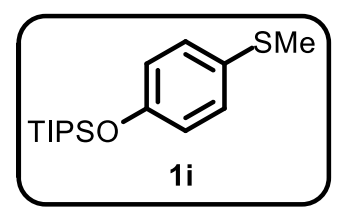

\section{Triisopropyl(4-methylsulfanylphenoxy)silane (1i):}


Colorless liquid; ${ }^{1} \mathrm{H}$ NMR $\left(\mathrm{CDCl}_{3}\right) \delta 7.19(\mathrm{~d}, J=9.0 \mathrm{~Hz}, 2 \mathrm{H}), 7.82(\mathrm{~d}, J=9.0 \mathrm{~Hz}, 2 \mathrm{H}), 2.44$ $(\mathrm{s}, 3 \mathrm{H}), 1.27-1.22(\mathrm{~m}, 3 \mathrm{H}), 1.10(\mathrm{~d}, J=7.8 \mathrm{~Hz}, 18 \mathrm{H}) \mathrm{ppm} ;{ }^{13} \mathrm{C} \mathrm{NMR}\left(\mathrm{CDCl}_{3}\right) \delta 154.5,129.7$, 129.0, 120.5, 17.9, 17.8, 12.6 ppm; HRMS (APCI-MS, Positive) $(\mathrm{m} / \mathrm{z}):[\mathrm{M}]^{+}$Calcd for $\mathrm{C}_{16} \mathrm{H}_{28} \mathrm{OSSi}$, 296.1630; found, 296.1627.

\section{Synthesis of 4-(dimethoxymethyl)phenyl methyl sulfide (1u): ${ }^{10}$}

To a solution of 4-methylsulfanylbenzaldehyde $(761 \mathrm{mg}, 5.0 \mathrm{mmol})$ in anhydrous $\mathrm{MeOH}$ (20 $\mathrm{mL}$ ) was added pyridinium $p$-toluenesulfonate $(63 \mathrm{mg}, 0.25 \mathrm{mmol})$ under argon atmosphere. The resulting solution was refluxed for $18 \mathrm{~h}$. The reaction mixture was quenched with sat. $\mathrm{NaHCO}_{3}$ solution $(3 \mathrm{~mL})$ and extracted with ethyl acetate $(3 \times 20 \mathrm{~mL})$. The organic layer was separated, dried over $\mathrm{Na}_{2} \mathrm{SO}_{4}$, and concentrated under vacuum. The crude residue was purified using column chromatography on silica gel $($ Hexane/EtOAc $=19 / 1)$ to provide 4(dimethoxymethyl)phenyl methyl sulfide (1u, $900 \mathrm{mg}, 4.54 \mathrm{mmol}, 91 \%$ yield).

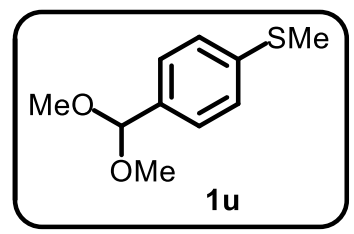

\section{4-(Dimethoxymethyl)phenyl methyl sulfide (1u):}

Colorless liquid; ${ }^{1} \mathrm{H} \mathrm{NMR}\left(\mathrm{CDCl}_{3}\right) \delta 7.36(\mathrm{~d}, J=9.0 \mathrm{~Hz}, 2 \mathrm{H}), 7.25(\mathrm{~d}, J=9.0 \mathrm{~Hz}, 2 \mathrm{H}), 5.36$ $(\mathrm{s}, 1 \mathrm{H}), 3.31(\mathrm{~s}, 6 \mathrm{H}), 2.49(\mathrm{~s}, 3 \mathrm{H}) \mathrm{ppm} ;{ }^{13} \mathrm{C} \mathrm{NMR}\left(\mathrm{CDCl}_{3}\right) \delta 138.7,134.9,127.1,126.1$, 102.7, 52.6, 15.7 ppm; HRMS (APCI-MS, Positive) $(\mathrm{m} / \mathrm{z})$ : $[\mathrm{M}]^{+}$Calcd for $\mathrm{C}_{10} \mathrm{H}_{14} \mathrm{O}_{2} \mathrm{~S}$, 198.0715; found, 198.0712.

\section{Synthesis of 2,5,5-trimethyl-2-(4-methylsulfanylphenyl)-1,3-dioxane (1v): ${ }^{11}$}

To a mixture of 4'-methylsulfanylacetophenone $(831 \mathrm{mg}, 5.0 \mathrm{mmol})$, trimethyl orthoformate (1.19 g, $7.5 \mathrm{mmol})$, and neopentyl glycol (1.04 g, $10.0 \mathrm{mmol})$ was added $\mathrm{In}(\mathrm{OTf})_{3}(28 \mathrm{mg}$, $0.05 \mathrm{mmol}$ ) under argon atmosphere. The resulting solution was stirred at room temperature for $1 \mathrm{~h}$. The reaction mixture was quenched with sat. $\mathrm{NaHCO}_{3}$ solution $(3 \mathrm{~mL})$ and extracted with ethyl acetate $(3 \times 20 \mathrm{~mL})$. The organic layer was separated, dried over $\mathrm{Na}_{2} \mathrm{SO}_{4}$, and concentrated under vacuum. The crude residue was purified using column chromatography on silica gel (Hexane/EtOAc $=9 / 1)$ to provide 2,5,5-trimethyl-2-(4-methylsulfanylphenyl)1,3-dioxane (1v, $1.10 \mathrm{~g}, 4.36 \mathrm{mmol}, 87 \%$ yield). 


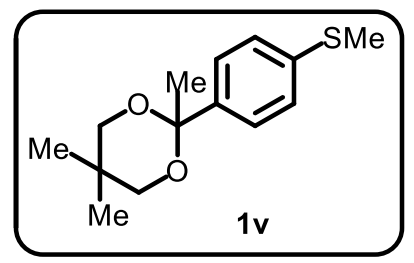

\section{2,5,5-Trimethyl-2-(4-methylsulfanylphenyl)-1,3-dioxane (1v):}

Colorless solid. $\mathrm{mp}=69-70{ }^{\circ} \mathrm{C} ;{ }^{1} \mathrm{H} \mathrm{NMR}\left(\mathrm{CDCl}_{3}\right) \delta 7.34(\mathrm{~d}, J=9.0 \mathrm{~Hz}, 2 \mathrm{H}), 7.27(\mathrm{~d}, J=$ $9.0 \mathrm{~Hz}, 2 \mathrm{H}), 3.42(\mathrm{~d}, J=10.2 \mathrm{~Hz}, 2 \mathrm{H}), 3.36(\mathrm{~d}, J=10.2 \mathrm{~Hz}, 2 \mathrm{H}), 2.51(\mathrm{~s}, 3 \mathrm{H}), 1.52(\mathrm{~s}, 3 \mathrm{H})$, $1.26(\mathrm{~s}, 3 \mathrm{H}), 0.58(\mathrm{~s}, 3 \mathrm{H}) \mathrm{ppm} ;{ }^{13} \mathrm{C} \mathrm{NMR}\left(\mathrm{CDCl}_{3}\right) \delta$ 137.8, 137.7, 127.3, 126.6, 100.0, 71.7, 31.9, 29.9, 22.8, 21.9, 15.7 ppm; HRMS (APCI-MS, Positive) $(\mathrm{m} / \mathrm{z}):[\mathrm{M}+\mathrm{H}]^{+}$Calcd for $\mathrm{C}_{14} \mathrm{H}_{21} \mathrm{O}_{2} \mathrm{~S}, 253.1257$; found, 253.1255.

\section{General Procedure for Palladium-Catalyzed Borylation of Aryl Sulfides:}

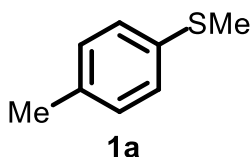

1a

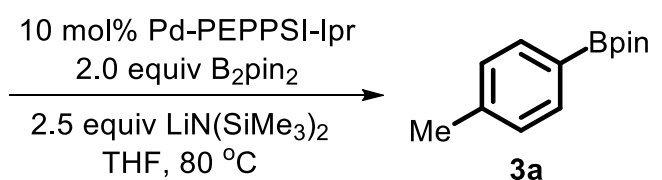

3a

The preparation of $\mathbf{3 a}$ is representative. 4-Methylthioanisole (1a, $69 \mathrm{mg}, 0.5 \mathrm{mmol}), \mathrm{B}_{2} \mathrm{pin}_{2}$ (254 mg, $1.0 \mathrm{mmol}), \mathrm{LiN}\left(\mathrm{SiMe}_{3}\right)_{2}(209 \mathrm{mg}, 1.25 \mathrm{mmol})$, and Pd-PEPPSI-IPr (34 mg, 0.05 mmol) were taken in an oven-dried Schlenk tube (with a screw cap) under an argon atmosphere. THF $(2.0 \mathrm{~mL})$ was added to this mixture. The resulting mixture was stirred at 80 ${ }^{\circ} \mathrm{C}$ for $15 \mathrm{~h}$. The reaction mixture was then cooled to room temperature, quenched with sat. $\mathrm{NH}_{4} \mathrm{Cl}$ solution $(3 \mathrm{~mL})$, and extracted with ethyl acetate $(3 \times 20 \mathrm{~mL})$. The combined organic layer was separated, dried over $\mathrm{Na}_{2} \mathrm{SO}_{4}$, and concentrated under vacuum. The crude residue was purified by using column chromatography on B-silica gel (Hexane/EtOAc $=49 / 1)$ to provide 4,4,5,5-tetramethyl-2-(4-tolyl)-1,3,2-dioxaborolane (3a, $88 \mathrm{mg}, 0.40 \mathrm{mmol}, 81 \%$ yield) as a colorless solid. ${ }^{13}$

Compound 3b: Yellows oil (88 mg, $0.43 \mathrm{mmol}, 86 \%) .^{13}$

Compound 3c: Purified by using column chromatography on silica gel [Kanto, $60 \mathrm{~N}$ (neutral, $63-210 \mu \mathrm{m})$ ] eluting with $\mathrm{Hexane} / \mathrm{Et}_{2} \mathrm{O}=30 / 1$. Yellows oil $(46 \mathrm{mg}, 0.20 \mathrm{mmol}, 39 \%){ }^{13}$

Compound 3d: Pale yellows solid ( $88 \mathrm{mg}, 0.36 \mathrm{mmol}, 71 \%) .^{13}$ 
Compound 3e: Yellows solid (161 mg, $0.44 \mathrm{mmol}, 87 \%) .{ }^{14}$

Compound 3f: Purified by using column chromatography on silica gel [Kanto, $60 \mathrm{~N}$ (neutral, 63-210 $\mu \mathrm{m})$ ] eluting with $\mathrm{DCM} / \mathrm{MeOH}=4 / 1$. Pale orange solid $(105 \mathrm{mg}, 0.36 \mathrm{mmol}, 72 \%) .{ }^{15}$

Compound 3g: Yellows solid (101 mg, $0.33 \mathrm{mmol}, 65 \%) .{ }^{16}$

Compound 3h: Pale yellow oil (88 mg, $0.33 \mathrm{mmol}, 67 \%) .{ }^{13}$

Compound 3i: Pale yellow solid (132 mg, $0.35 \mathrm{mmol}, 70 \%) .{ }^{13}$

Compound 3j: White solid (46 mg, $0.21 \mathrm{mmol}, 42 \%){ }^{17}$

Compound 3k: Orange solid (97 mg, $0.36 \mathrm{mmol}, 72 \%) .{ }^{13}$

Compound 31: Purified by using column chromatography on B-silica gel eluting with $\mathrm{DCM} / \mathrm{MeOH}=19 / 1$. Light brown solid (103 mg, $0.381 \mathrm{mmol}, 76 \%$ yield $) . \mathrm{mp}=80-81{ }^{\circ} \mathrm{C}$; ${ }^{1} \mathrm{H} \mathrm{NMR}\left(\mathrm{CDCl}_{3}\right) \delta$ 7.93-7.90 (m, 3H), $7.39(\mathrm{~d}, J=8.4 \mathrm{~Hz}, 2 \mathrm{H}), 7.32(\mathrm{t}, J=1.8 \mathrm{~Hz}, 1 \mathrm{H})$, $7.21(\mathrm{~s}, 1 \mathrm{H}), 1.36(\mathrm{~s}, 12 \mathrm{H}) \mathrm{ppm} ;{ }^{13} \mathrm{C} \mathrm{NMR}\left(\mathrm{CDCl}_{3}\right) \delta 139.5,136.5,135.4,130.6,120.2$, 117.9, 84.1, 24.9 ppm; HRMS (APCI-MS, Positive) (m/z): [M] $]^{+}$Calcd for $\mathrm{C}_{15} \mathrm{H}_{19} \mathrm{BN}_{2} \mathrm{O}_{2}$, 270.1540 ; found, 270.1549 .

Compound 3m: Yellow solid (117 mg, $0.42 \mathrm{mmol}, 83 \%) .{ }^{13}$

Compound 3n: Yellow solid (98 mg, $0.35 \mathrm{mmol}, 71 \%){ }^{18}$

Compound 3o: Yellow oil (94 mg, $0.43 \mathrm{mmol}, 86 \%) .{ }^{19}$

Compound 3p: Pale yellow solid (118 mg, $0.46 \mathrm{mmol}, 93 \%){ }^{20}$

Compound 3q: Colorless oil (77 mg, $0.35 \mathrm{mmol}, 71 \%){ }^{13}$

Compound 3r: Pale yellow solid (112 mg, $0.40 \mathrm{mmol}, 80 \%){ }^{13}$

Compound 3s: White solid (116 mg, $0.46 \mathrm{mmol}, 91 \%){ }^{21}$

Compound 3t: Yellow solid (67 mg, $0.25 \mathrm{mmol}, 49 \%) .{ }^{19}$

Compound 3u: Purified by using column chromatography on silica gel [Kanto, $60 \mathrm{~N}$ (neutral, 63-210 $\mu \mathrm{m})$ ] eluting with Hexane/EtOAc $=4 / 1$. Pale orange solid $(106 \mathrm{mg}, 0.38 \mathrm{mmol}$, $76 \%)^{22}$ 
Compound 3v: Purified by using column chromatography on silica gel [Kanto, $60 \mathrm{~N}$ (neutral, 63-210 $\mu \mathrm{m})$ ] eluting with Hexane/EtOAc $=4 / 1$. Pale yellow solid $(125 \mathrm{mg}, 0.38 \mathrm{mmol}, 75 \%$ yield). mp $=131-132{ }^{\circ} \mathrm{C} ;{ }^{1} \mathrm{H}$ NMR $\left(\mathrm{CDCl}_{3}\right) \delta 7.84(\mathrm{~d}, J=8.4 \mathrm{~Hz}, 2 \mathrm{H}), 7.44(\mathrm{~d}, J=8.4 \mathrm{~Hz}$, $2 \mathrm{H}), 3.43-3.35(\mathrm{~m}, 4 \mathrm{H}), 1.52(\mathrm{~s}, 3 \mathrm{H}), 1.35(\mathrm{~s}, 12 \mathrm{H}), 1.27$ (s, 3H), $0.56(\mathrm{~s}, 3 \mathrm{H}) \mathrm{ppm} ;{ }^{13} \mathrm{C} \mathrm{NMR}$ $\left(\mathrm{CDCl}_{3}\right) \delta 144.1,135.1,126.1,100.2,83.8,71.8,31.9,29.9,24.9,22.9,21.8$ ppm; HRMS (APCI-MS, Positive) $(\mathrm{m} / \mathrm{z})$ : $[\mathrm{M}]^{+}$Calcd for $\mathrm{C}_{19} \mathrm{H}_{29} \mathrm{BO}_{4}, 332.2159$; found, 332.2155.

Compound 4b: Yellow solid (39 mg, $0.19 \mathrm{mmol}, 38 \%){ }^{23}$

Compound 4c: Purified by using column chromatography on silica gel [Kanto, $60 \mathrm{~N}$ (neutral, 63-210 $\mu \mathrm{m})$ ] eluting with Hexane/Et $\mathrm{O}_{2} \mathrm{O}=50 / 1$ to $20 / 1$. Yellow oil $(41 \mathrm{mg}, 0.19 \mathrm{mmol}$, $38 \%)^{24}$ 


\section{References}

1. Hitosugi, S.; Tanimoto, D.; Nakanishi, W.; Isobe, H. Chem. Lett. 2012, 41, 972.

2. Liu, J.; Chen, G.; Xing, J.; Liao, J. Tetrahedron: Asymmetry 2011, 22, 575.

3. Luo, F.; Pan, C.; Li, L.; Chen, F.; Cheng, J. Chem. Commun. 2011, 47, 5304.

4. Xu, Y.; Cong, T.; Liu, P.; Sun, P. Org. Biomol. Chem. 2015, 13, 9742.

5. Joseph, P. J. A.; Priyadarshini, S.; Kantam, M. L.; Maheswaran, H. Catal. Sci. Technol. 2011, 1, 234.

6. Cabiddu, M. G.; Cabiddu, S.; Cadoni, E.; Montis, S. D.; Fattuoni, C.; Melis, S. Tetrahedron 2004, 60, 3915.

7. Guo, S.-R.; Yuan, Y.-Q. J. Chem.Res. 2009, 745.

8. Bhanuchandra, M.; Murakami, K.; Vasu, D.; Yorimitsu, H.; Osuka, A. Angew. Chem. Int. Ed. 2015, 54, 10234.

9. Green, T. W.; Wuts, P. G. M. Protective Groups in Organic Synthesis, 4th ed.; Wiley: New York, 2007.

10. Crimmins, M. T.; Siliphaivanh, P. Org. Lett. 2003, 5, 4641.

11. Smith, B. M.; Graham, A. E. Tetrahedron Lett. 2011, 52, 6281.

12. Morgan, K. F.; Hollingsworth, I. A.; Bull, J. A. Org. Biomol. Chem. 2015, 13, 5265.

13. Niwa, T.; Ochiai, H.; Watanabe, Y.; Hosoya, T. J. Am. Chem. Soc. 2015, 137, 14313.

14. Zhu, C.; Yamane, M. Org. Lett. 2012, 14, 4560.

15. Yamamoto, T.; Morita, T.; Takagi, J.; Yamakawa, T. Org. Lett. 2011, 13, 5766.

16. Ebisawa, M.; Ueno, M.; Oshima, Y.; Kondo, Y. Tetrahedron Lett. 2007, 48, 8918.

17. Qiu, D.; Jin, L.; Zheng, Z.; Meng, H.; Mo, F.; Wang, X.; Zhang, Y.; Wang, J. J. Org. Chem. 2013, 78, 1923.

18. Sakamoto, Y.; Suzuki, T. J. Am. Chem. Soc. 2013, 135, 14074.

19. Bose, S. K.; Deißenberger, A.; Eichhorn, A.; Steel, P. G.; Lin, Z.; Marder, T. B. Angew. Chem. Int. Ed. 2015, 54, 11843.

20. Miralles, N.; Romero, R. M.; Fernández, E.; Muñiz, K. Chem. Commun. 2015, 51, 14068.

21. Bose, S. K.; Marder, T. B. Org. Lett. 2014, 16, 4562.

22. Clary, J. W.; Rettenmaier, T. J.; Snelling, R.; Bryks, W.; Banwell, J.; Wipke, W. T.; Singaram, B. J. Org. Chem. 2011, 76, 9602.

23. Zhao, Y.; Snieckus, V. Org. Lett. 2014, 16, 3200.

24. PraveenGanesh, N.; Chavant, P. Y. Eur. J. Org. Chem. 2008, 4690. 
${ }^{1} \mathrm{H}$ and ${ }^{13} \mathrm{C}$ NMR Spectra
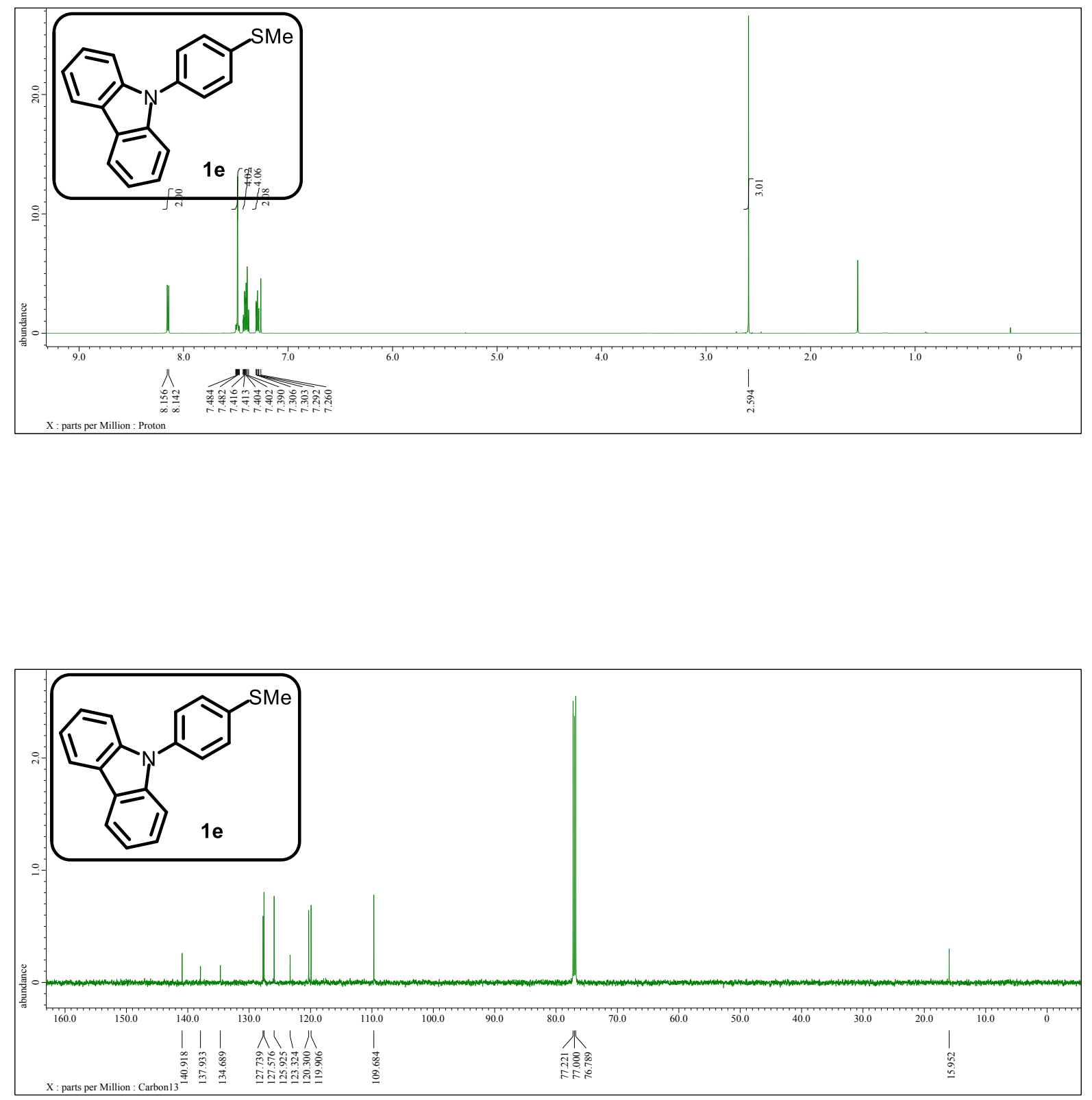

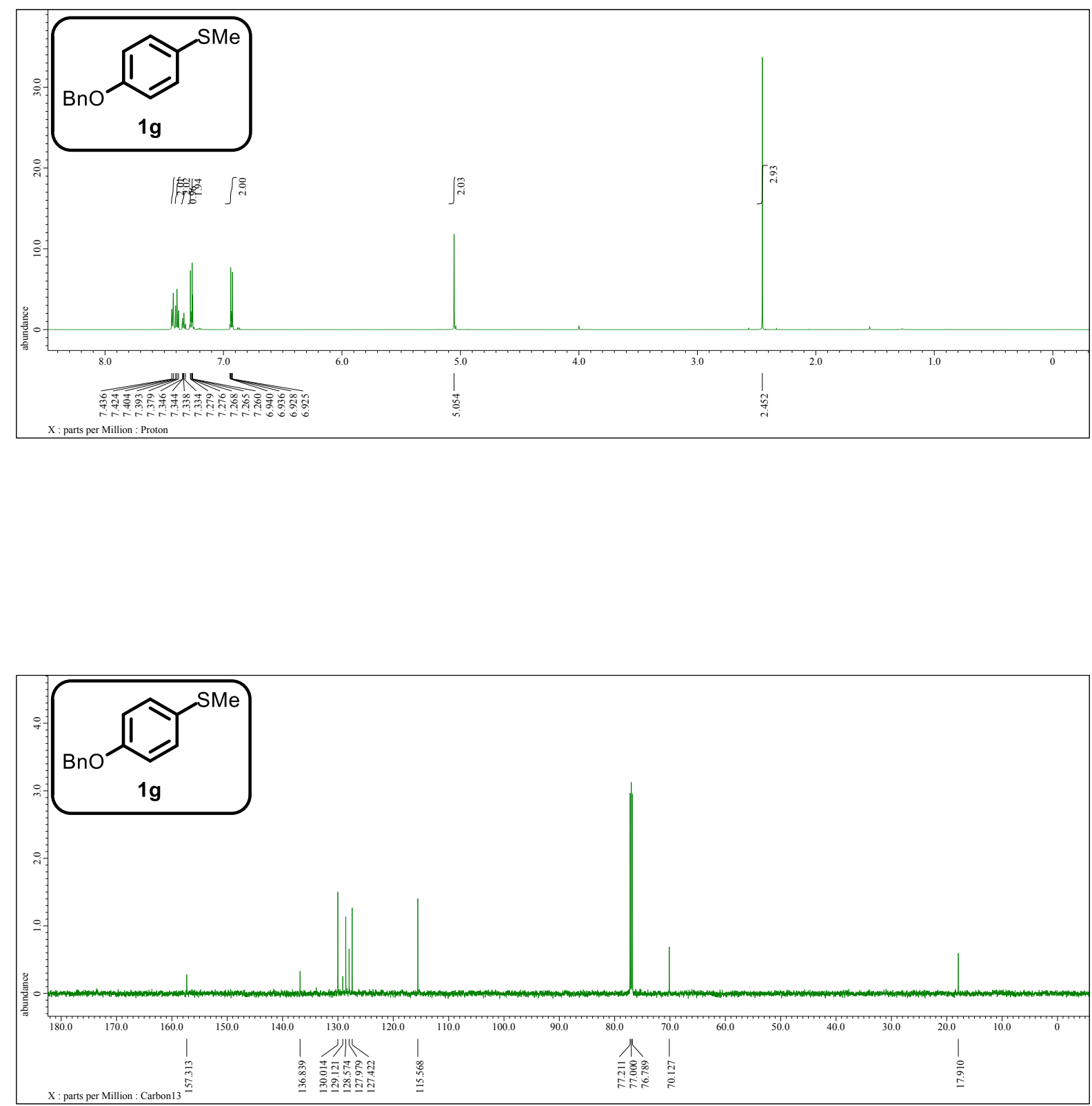

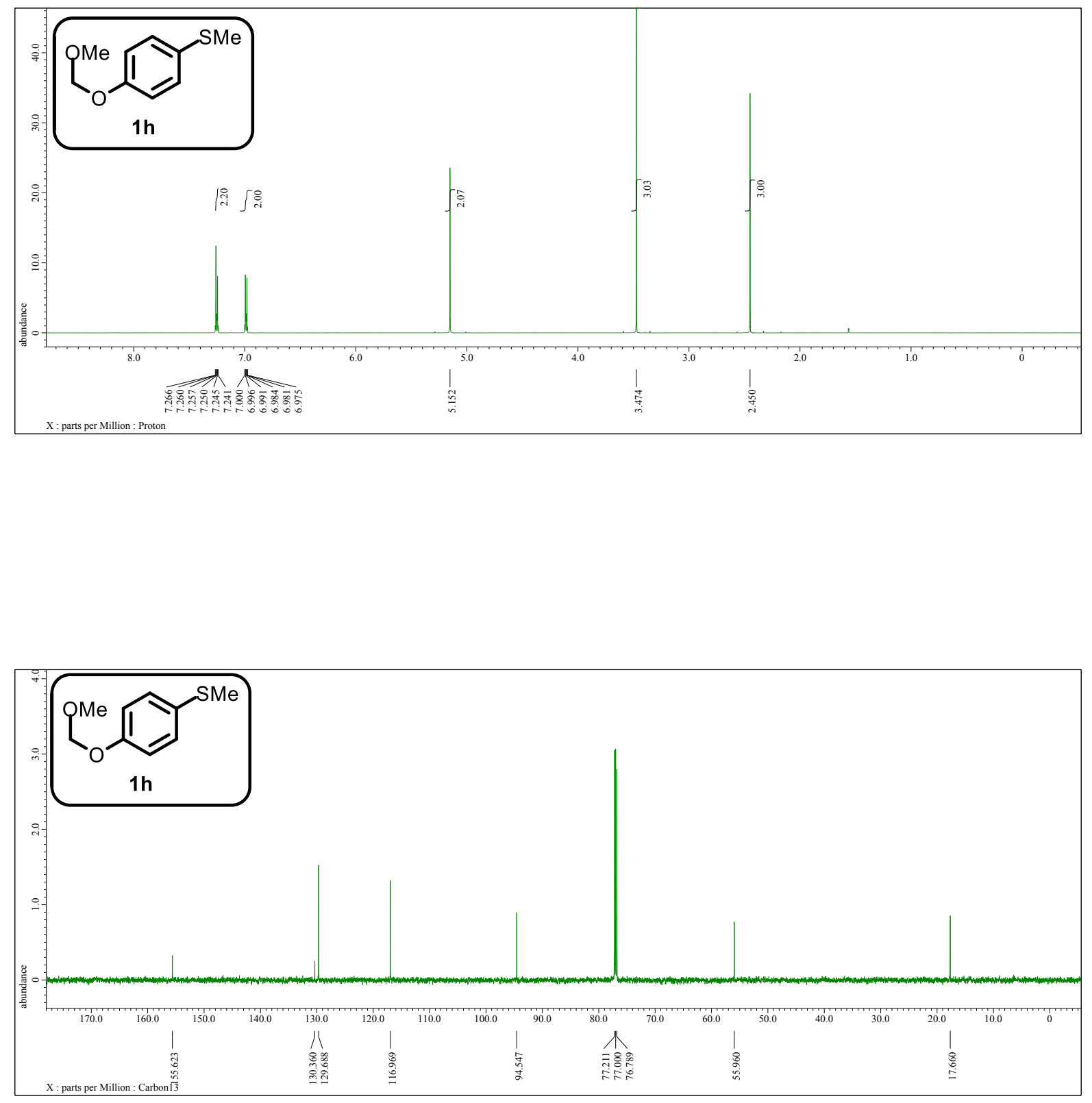

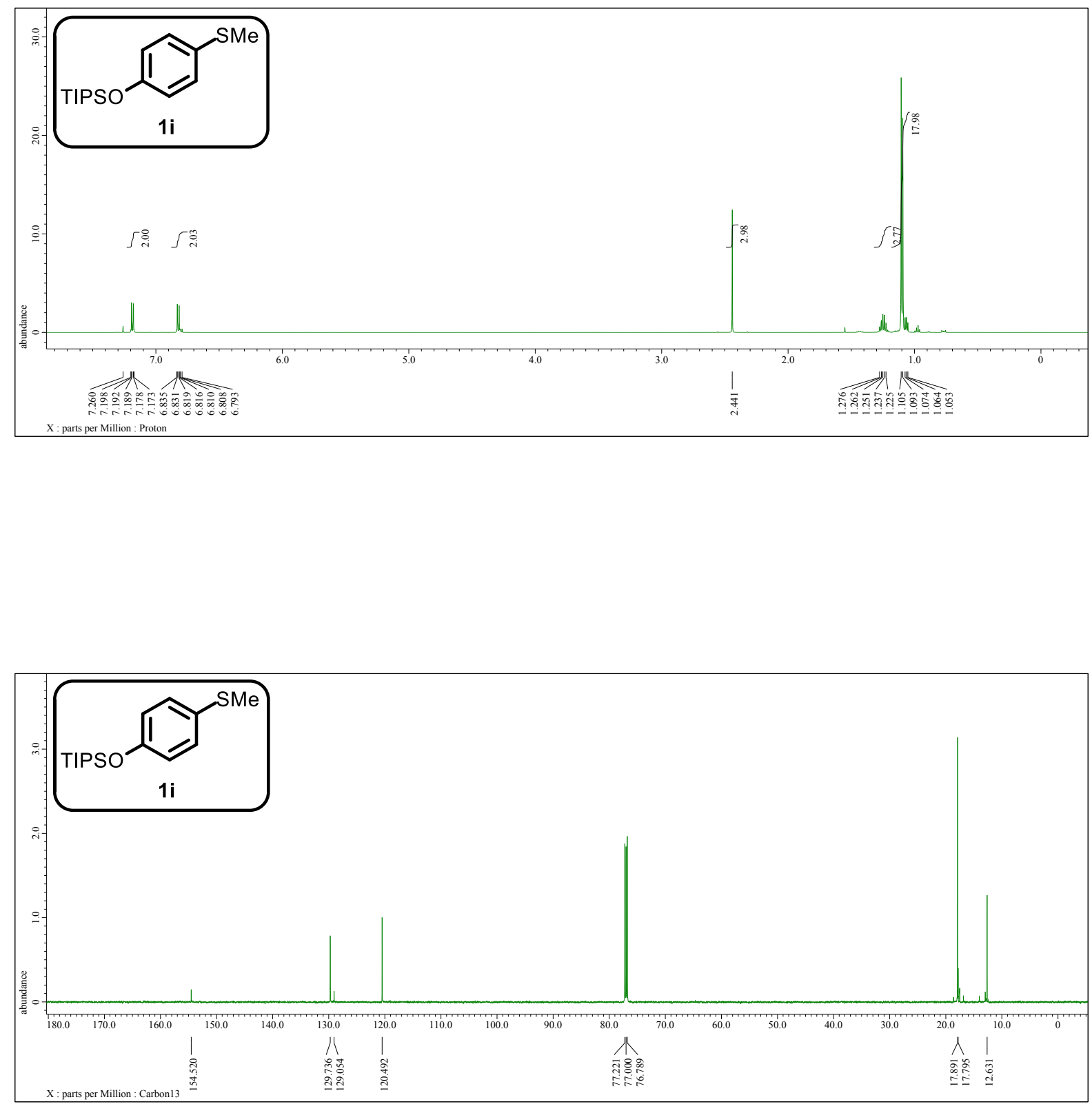

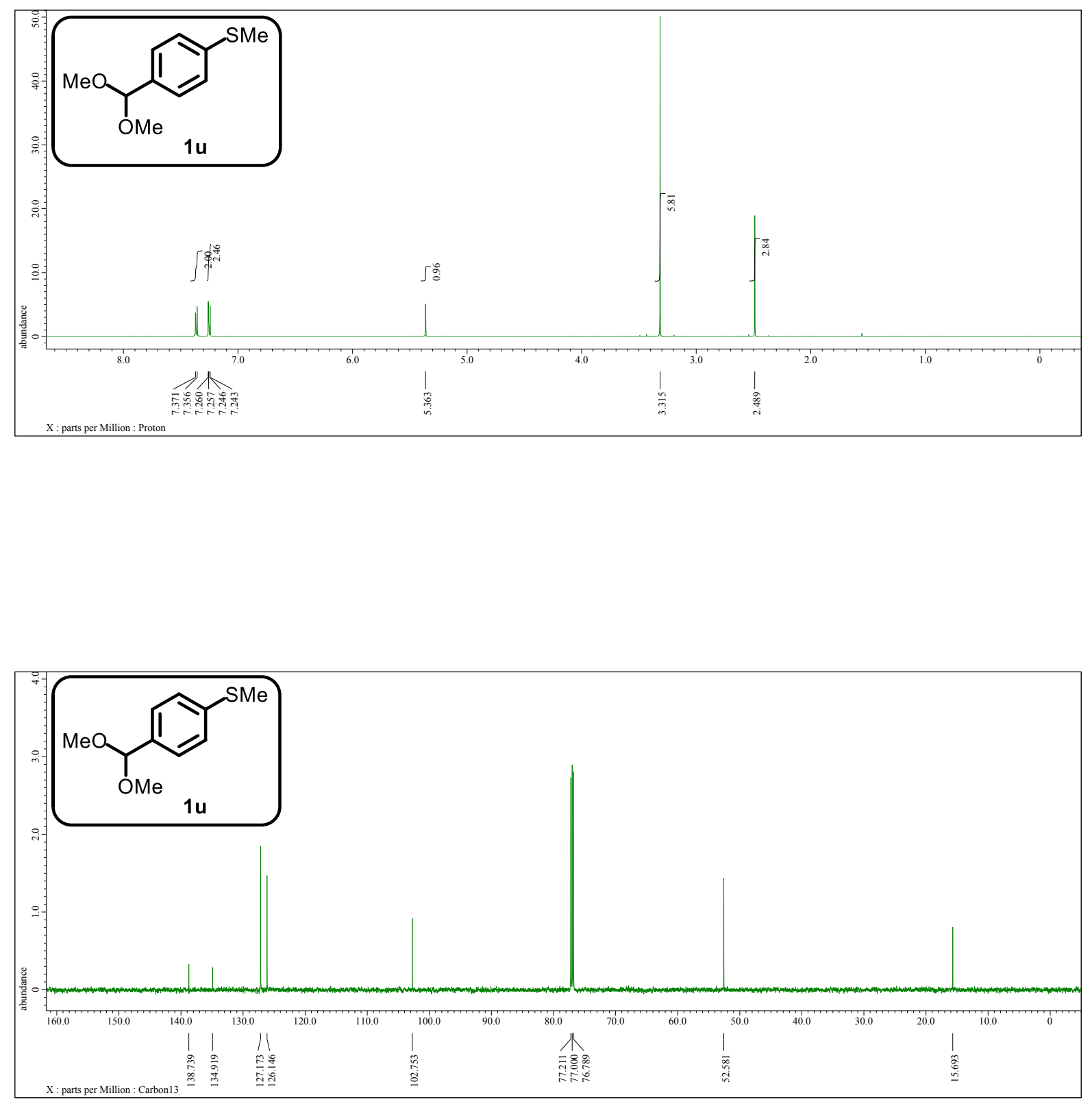

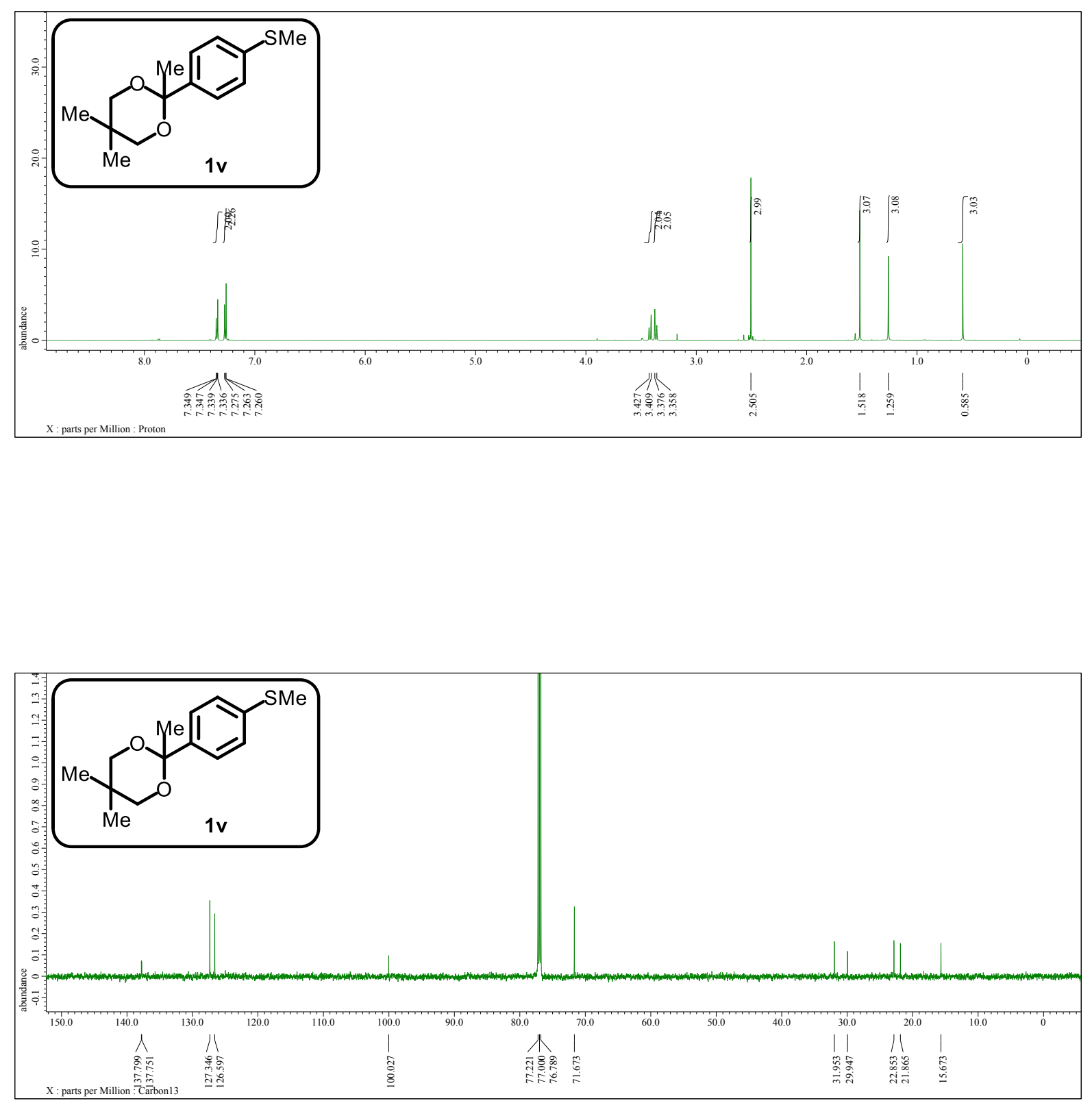

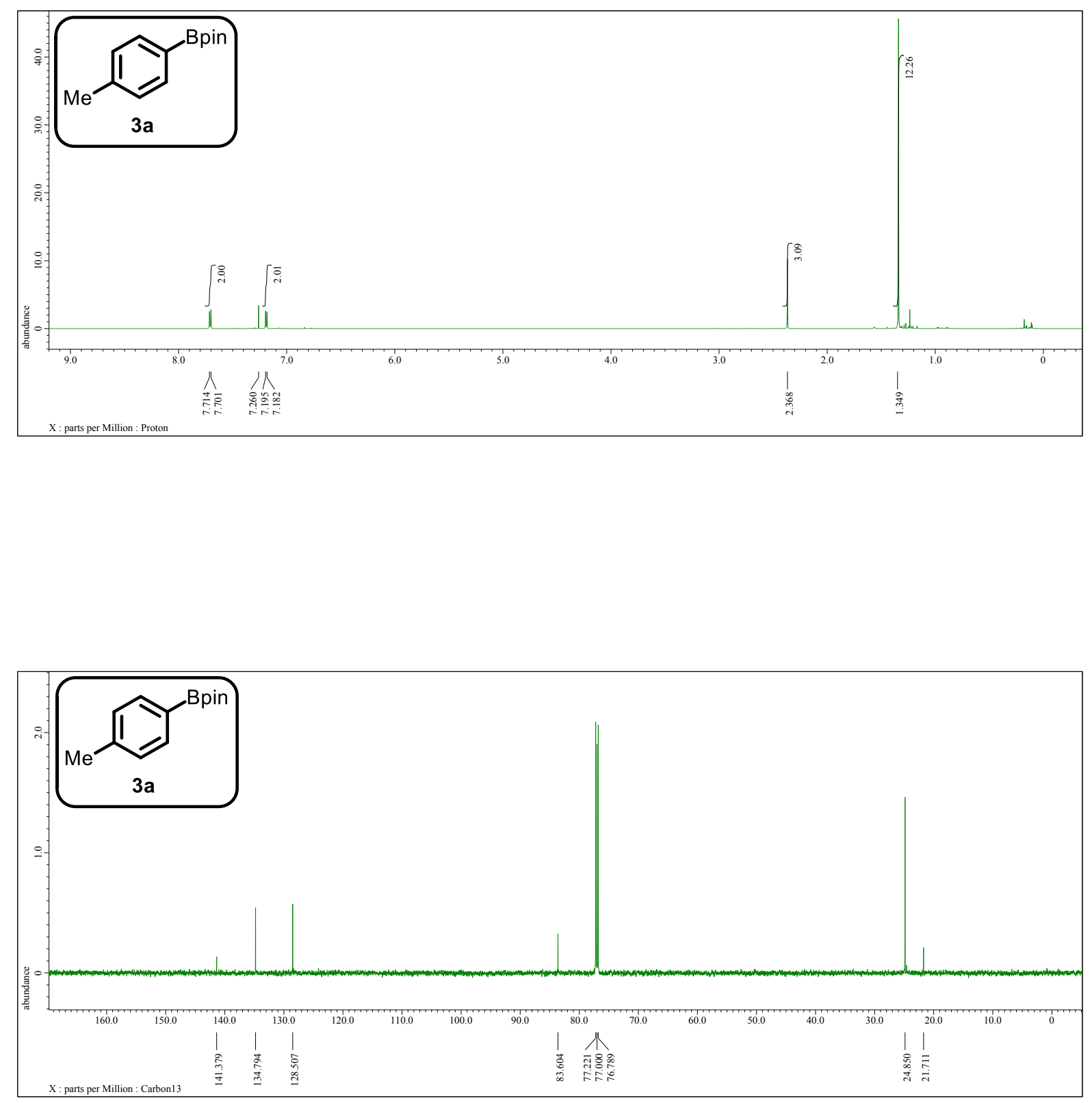

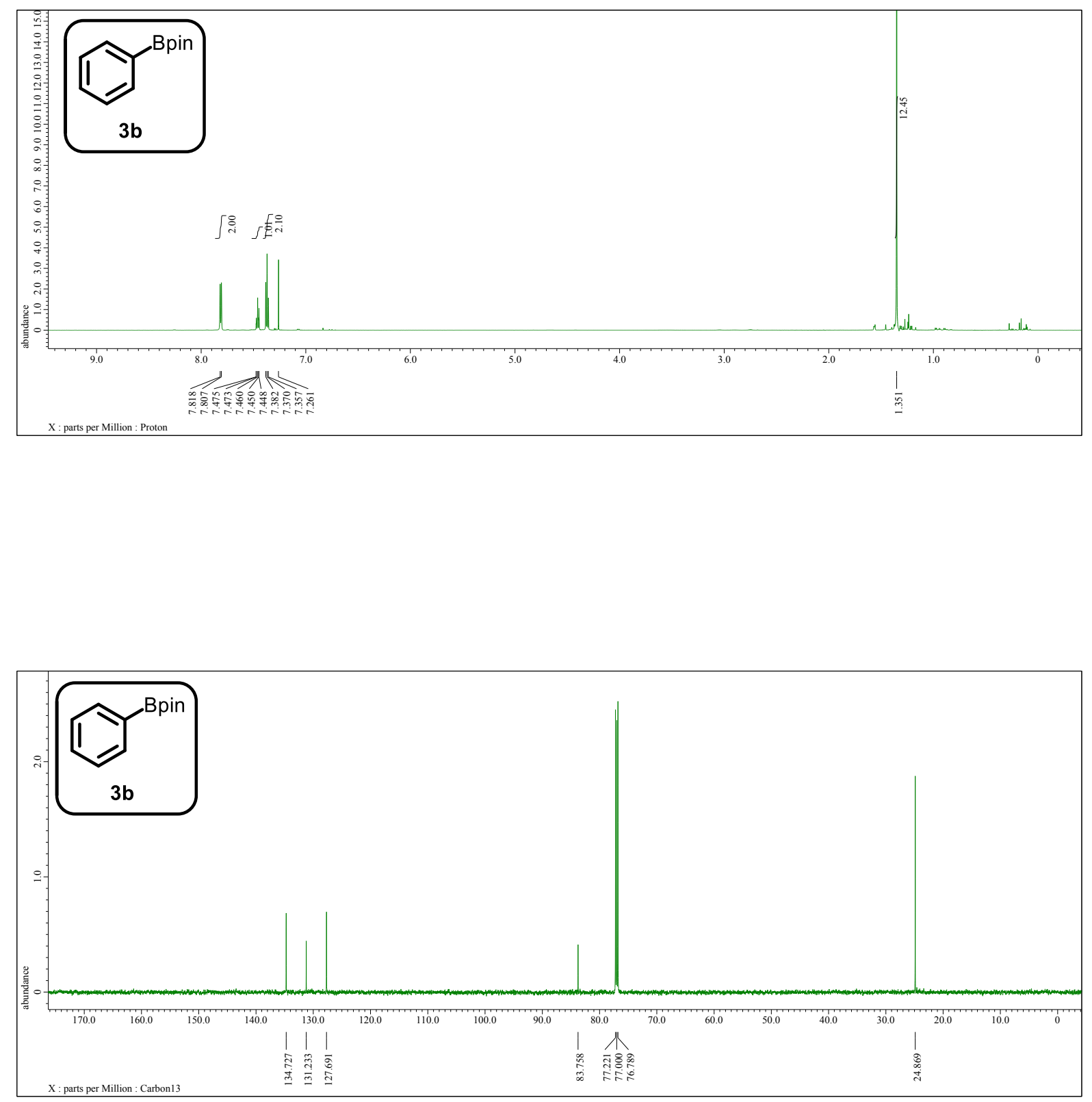

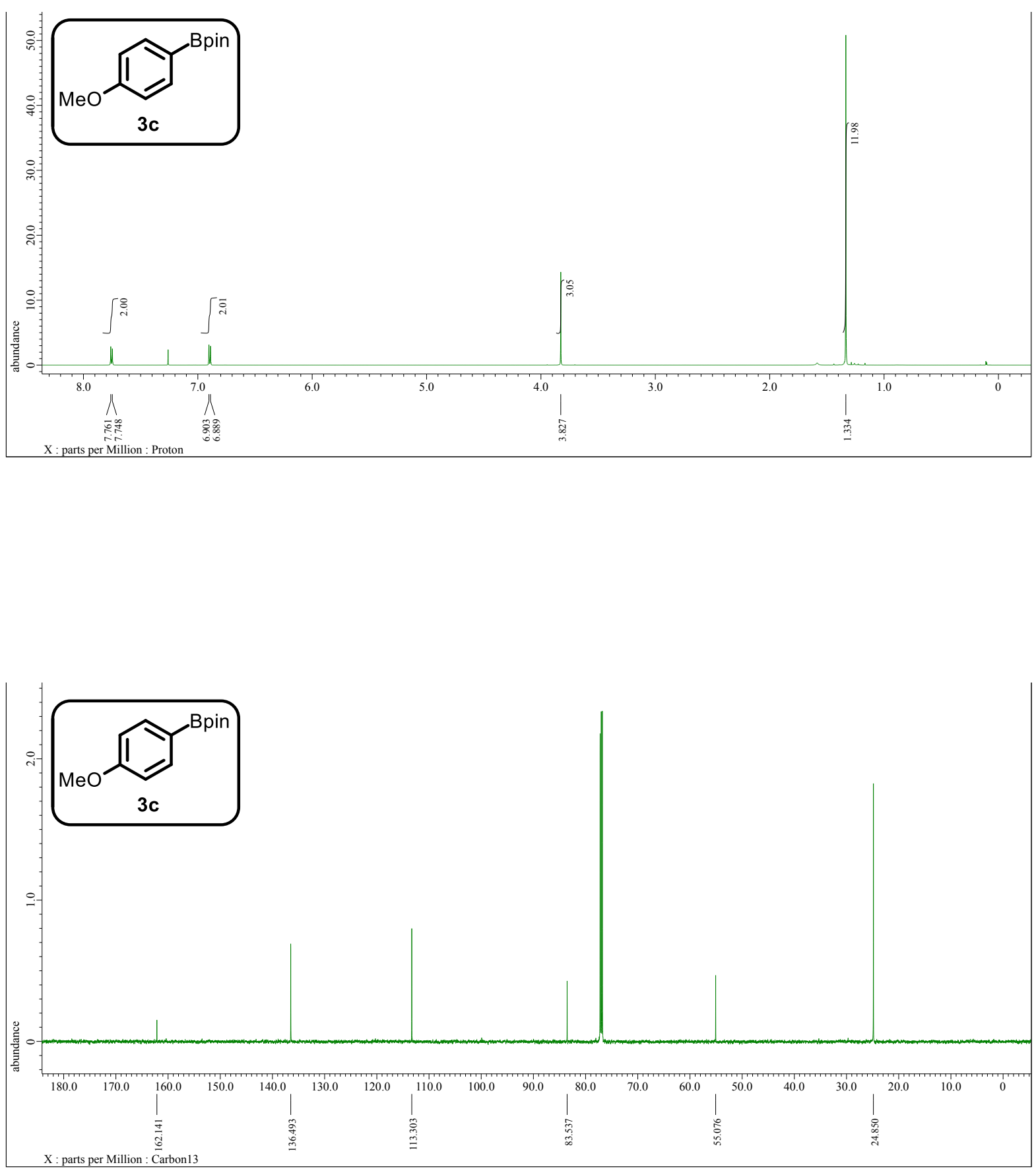

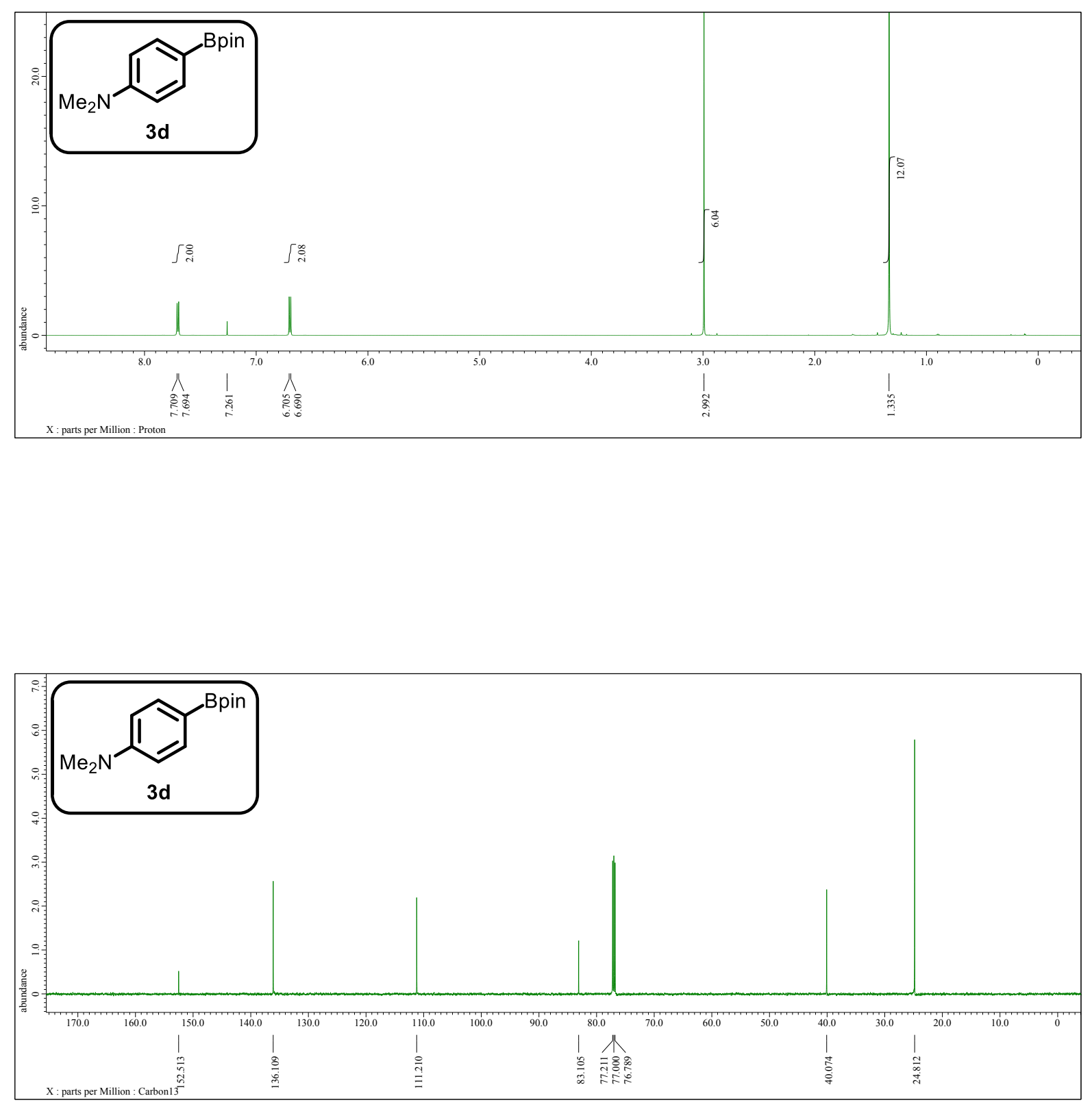

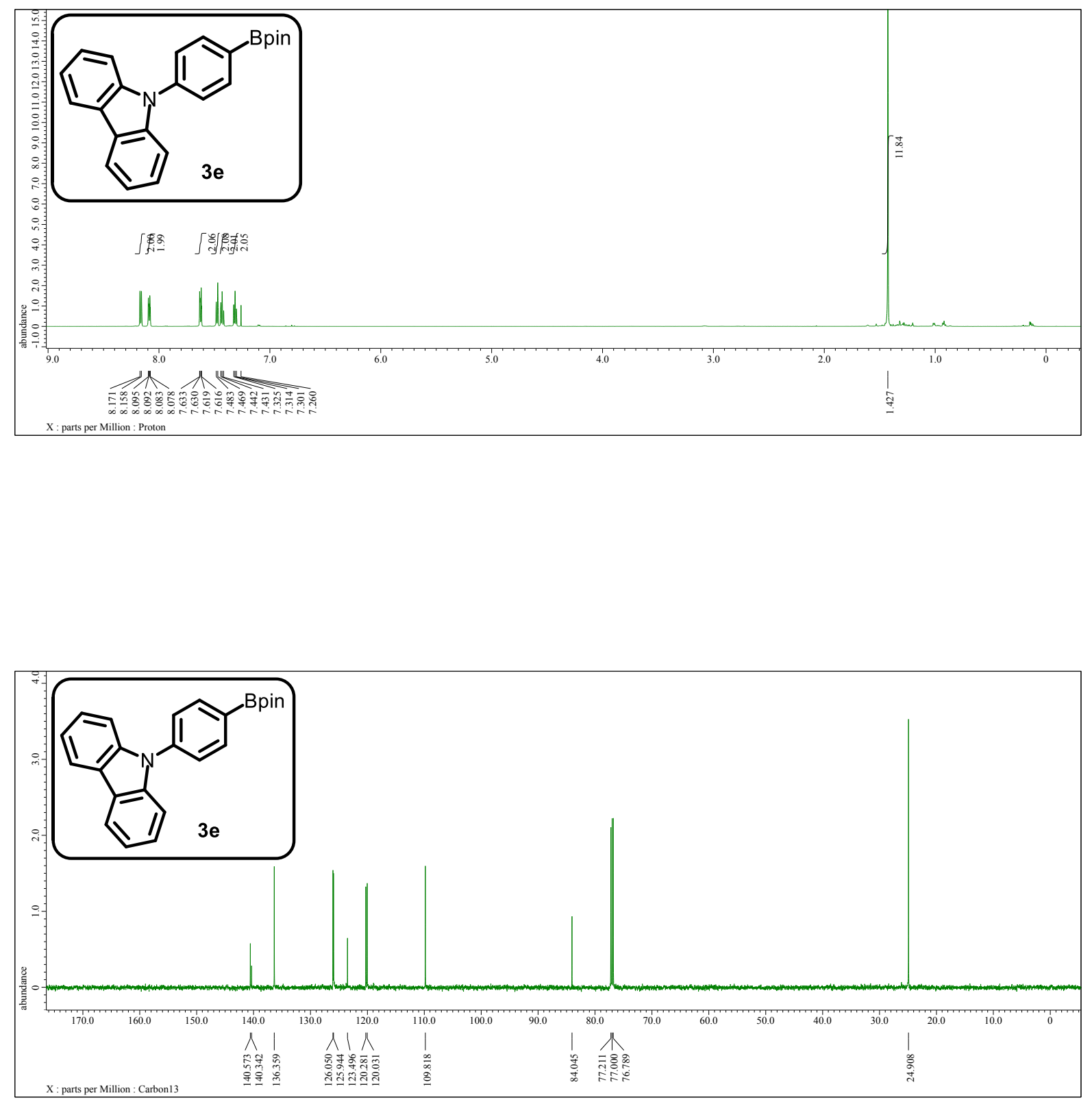

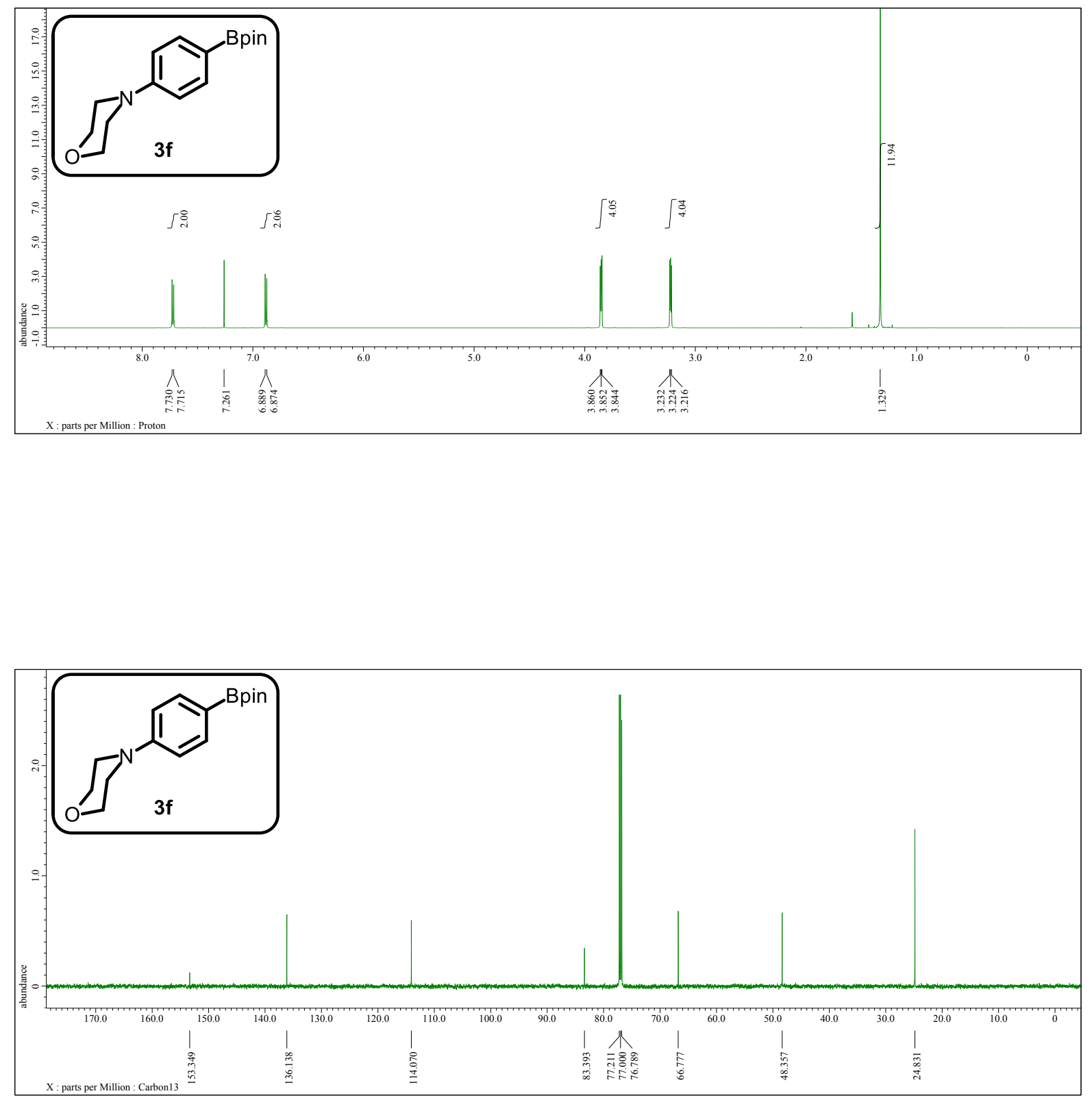

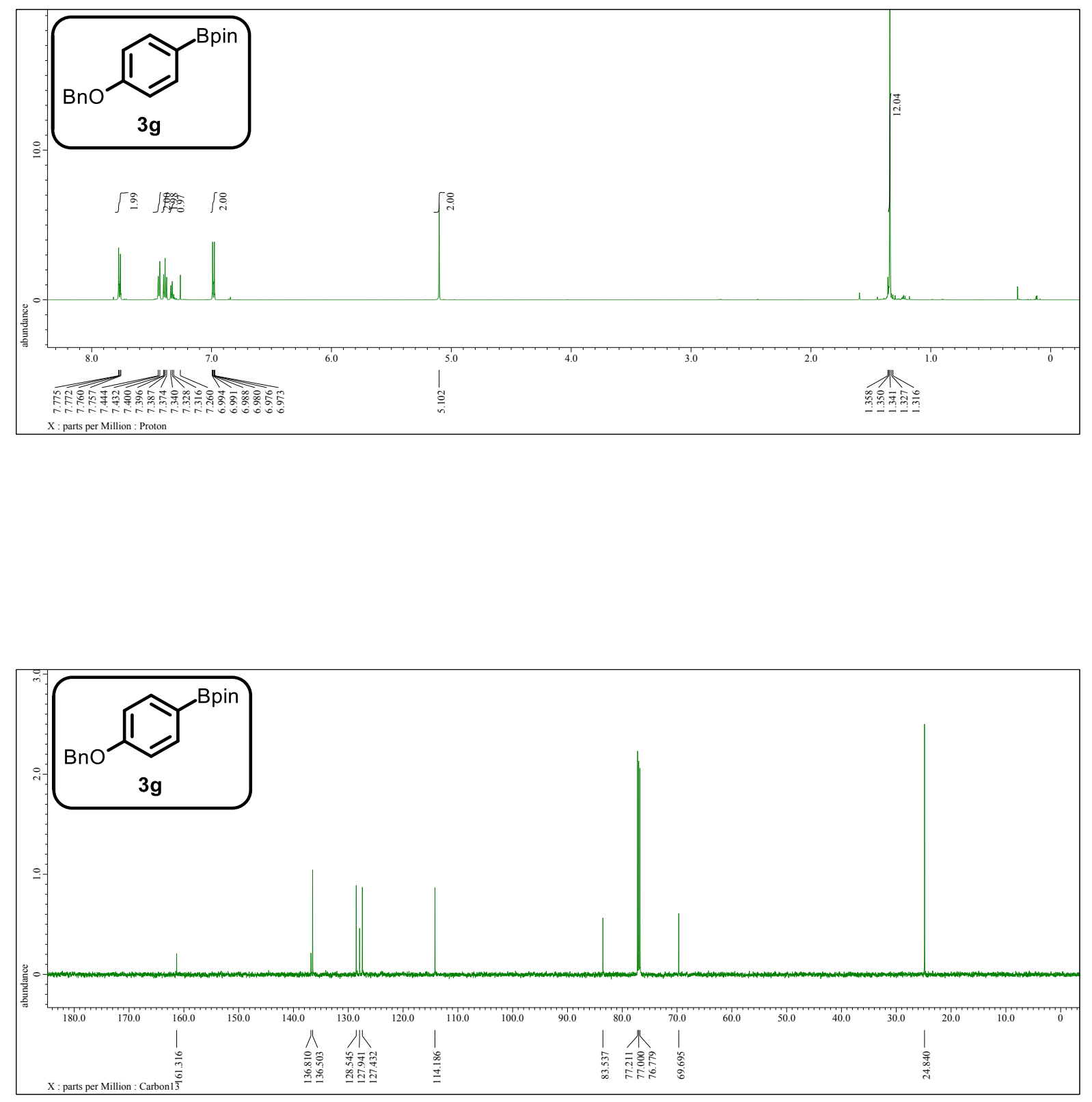

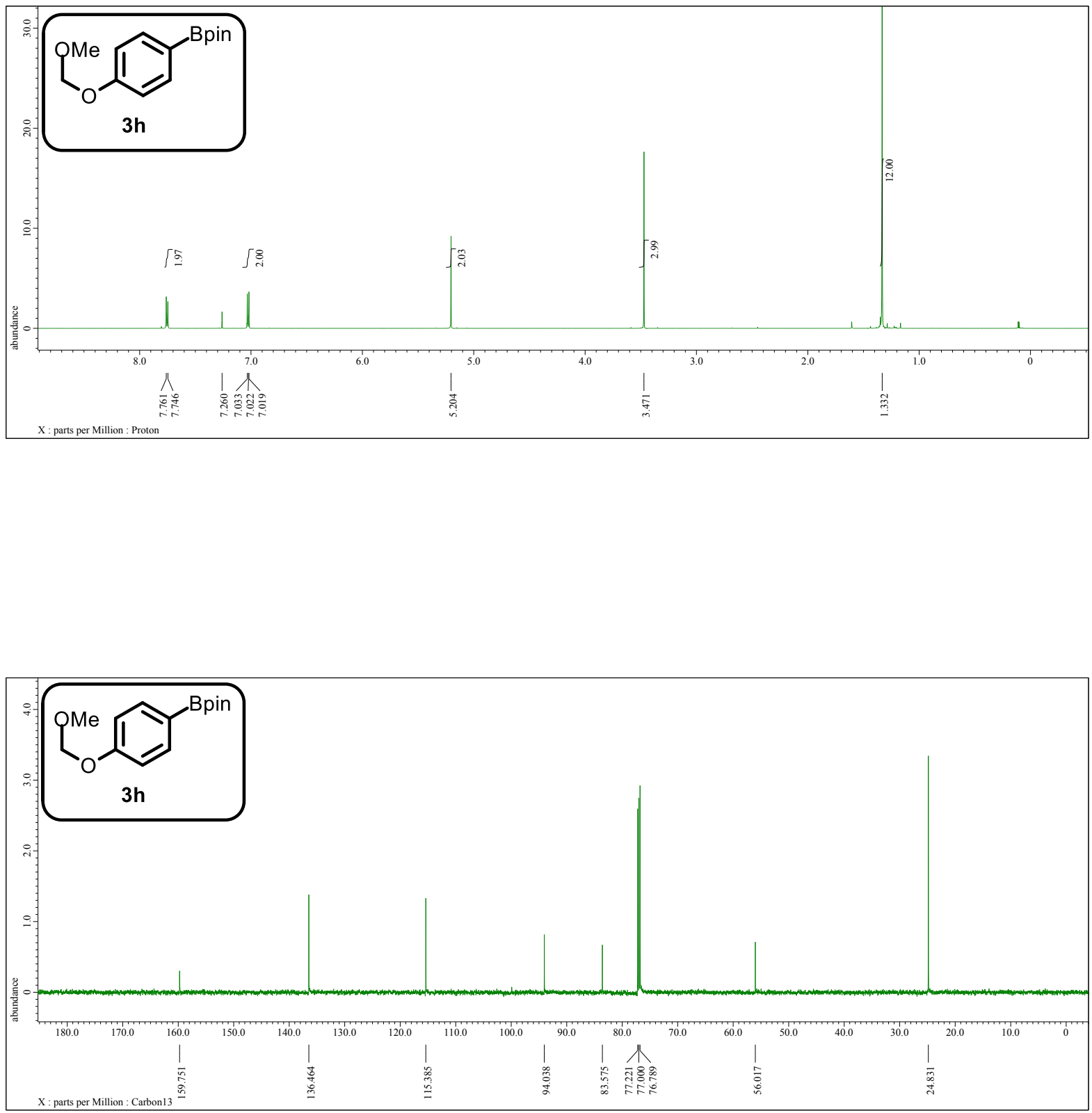

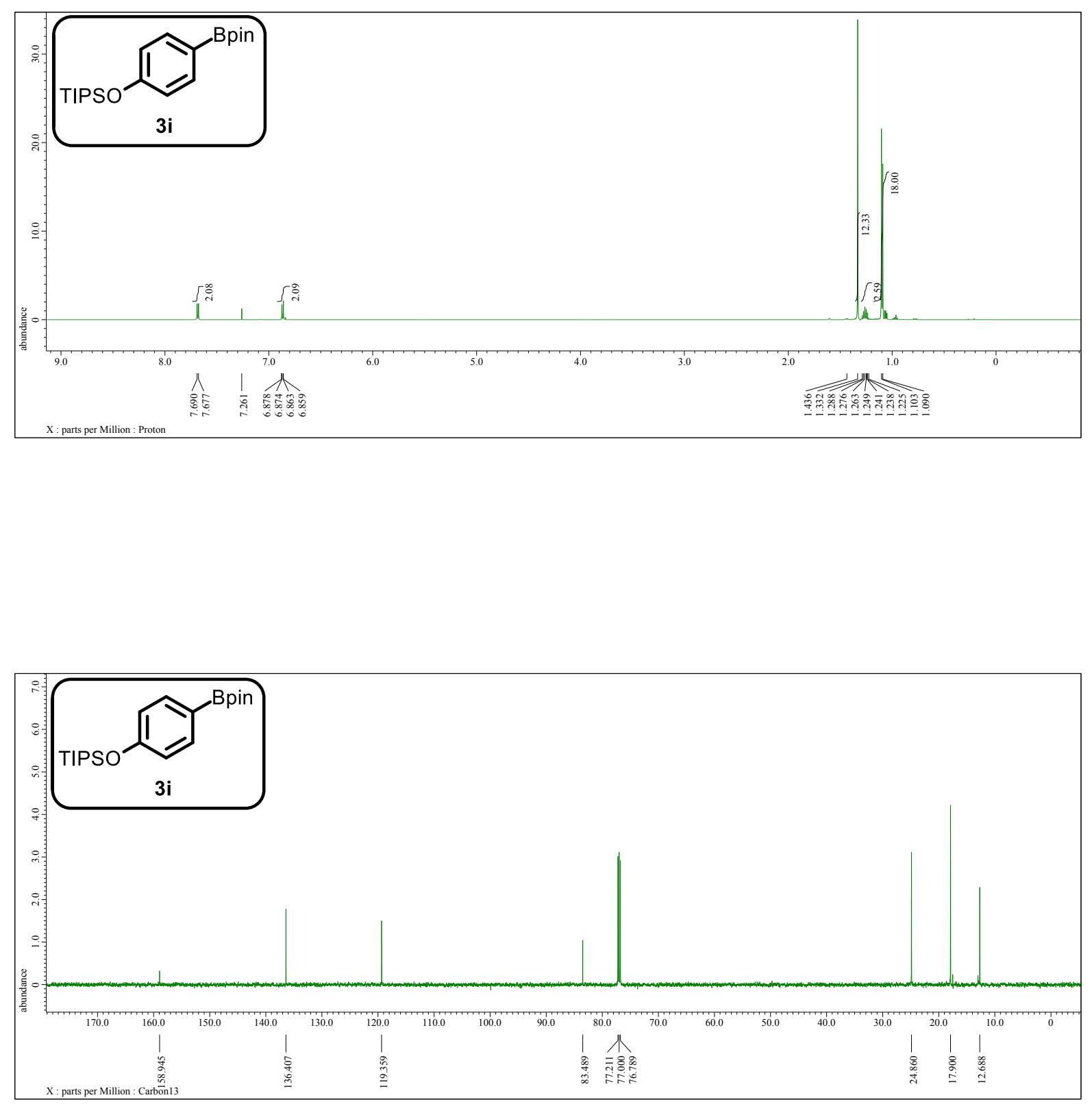

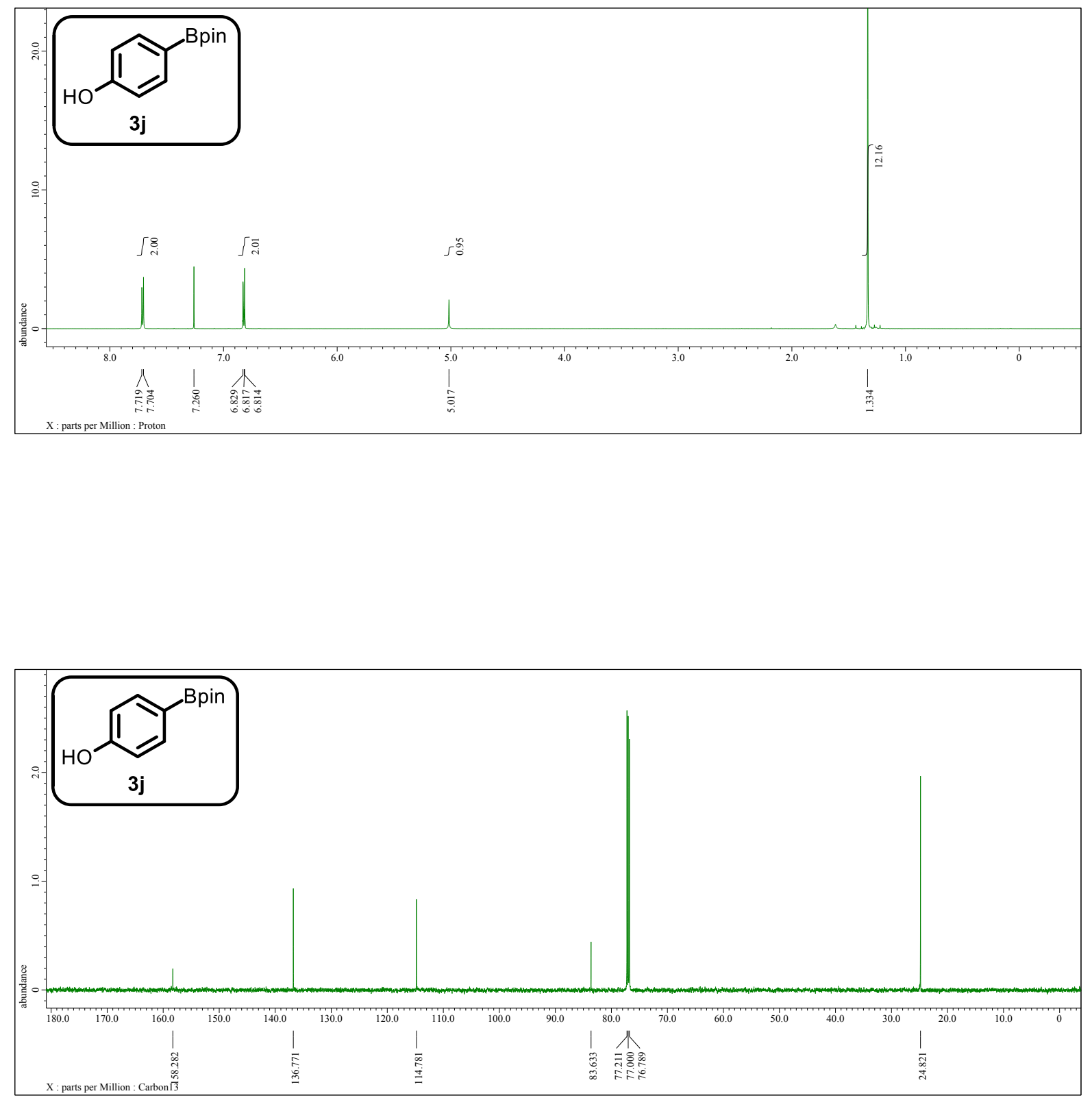

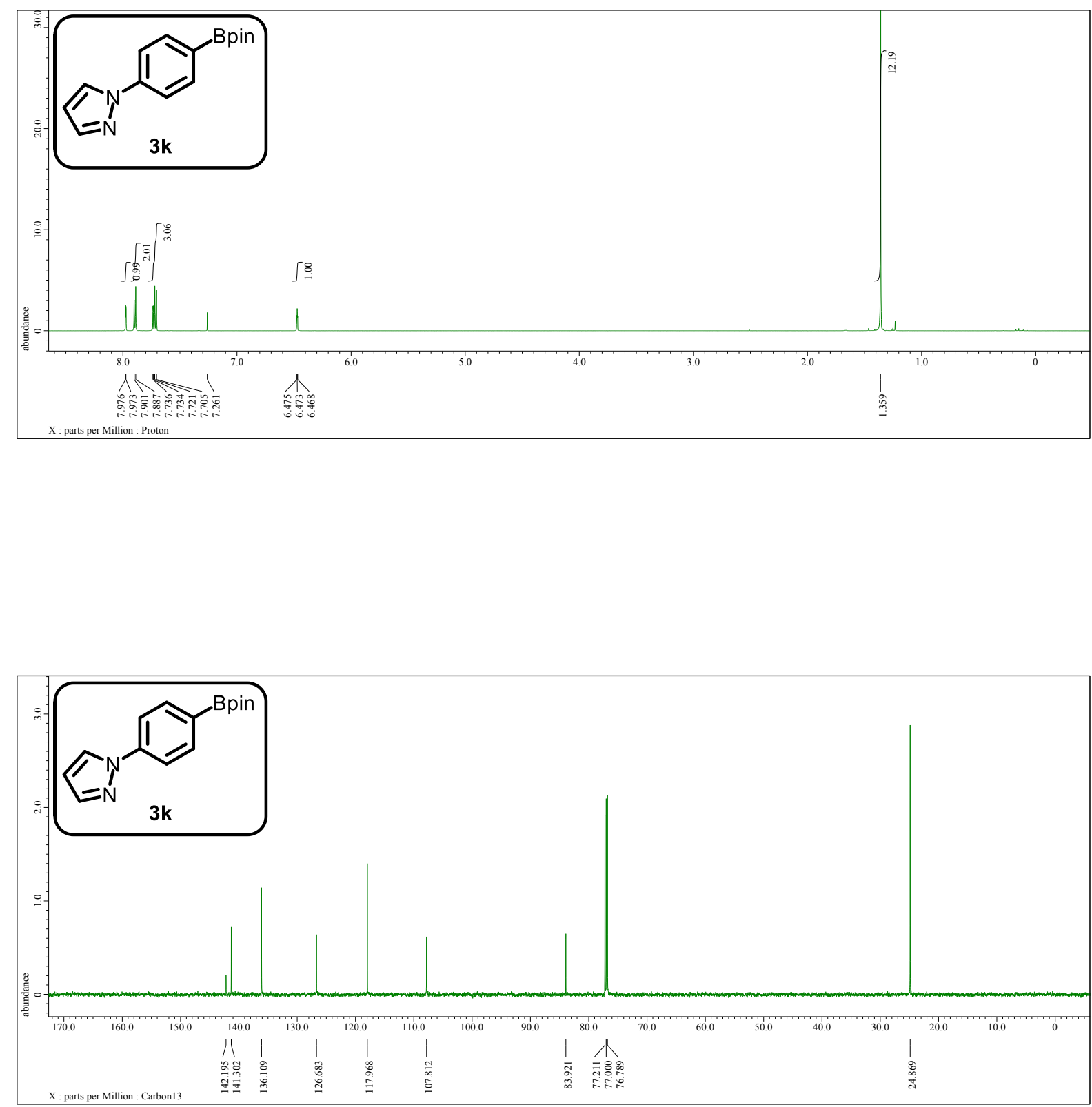

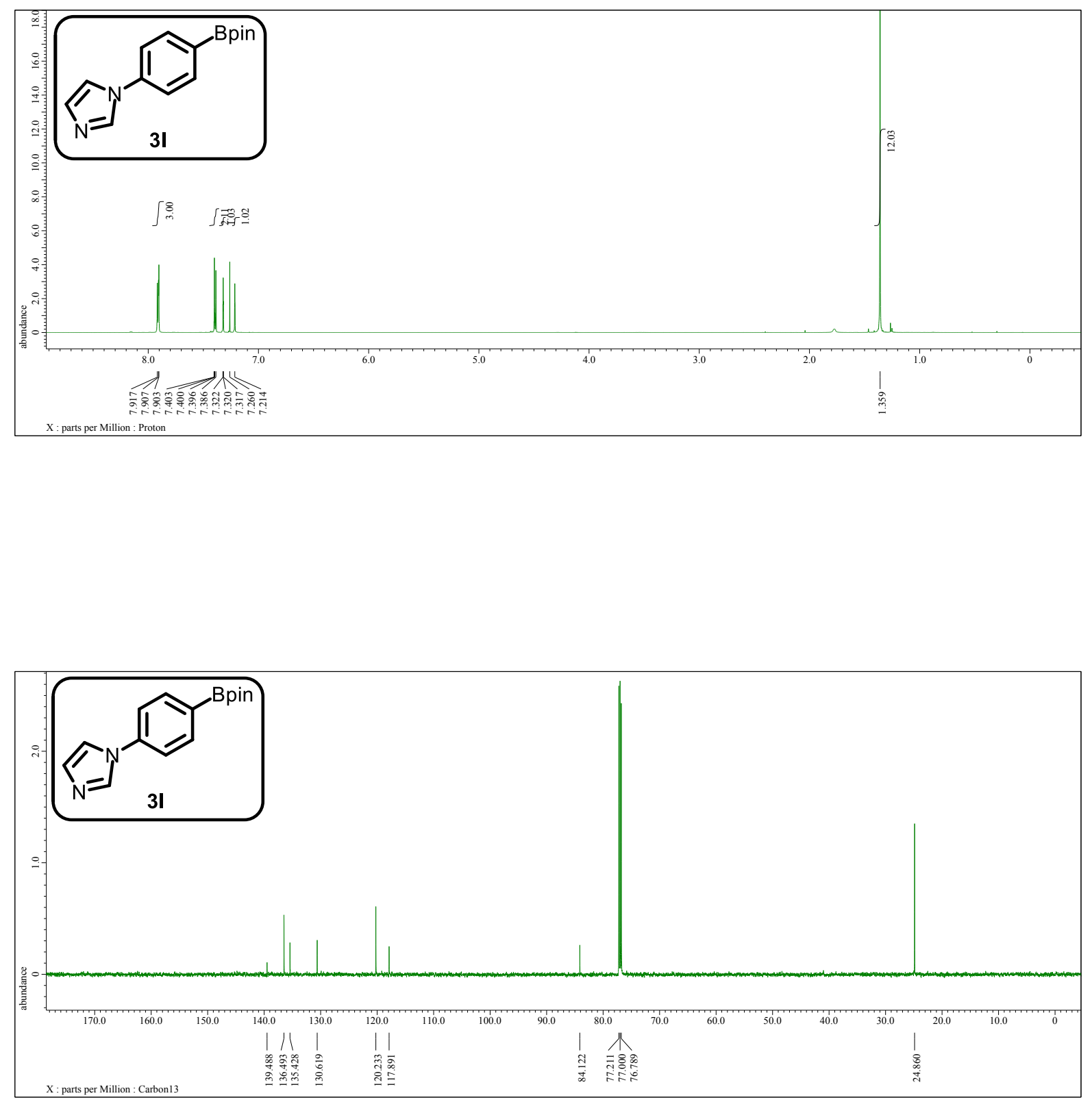

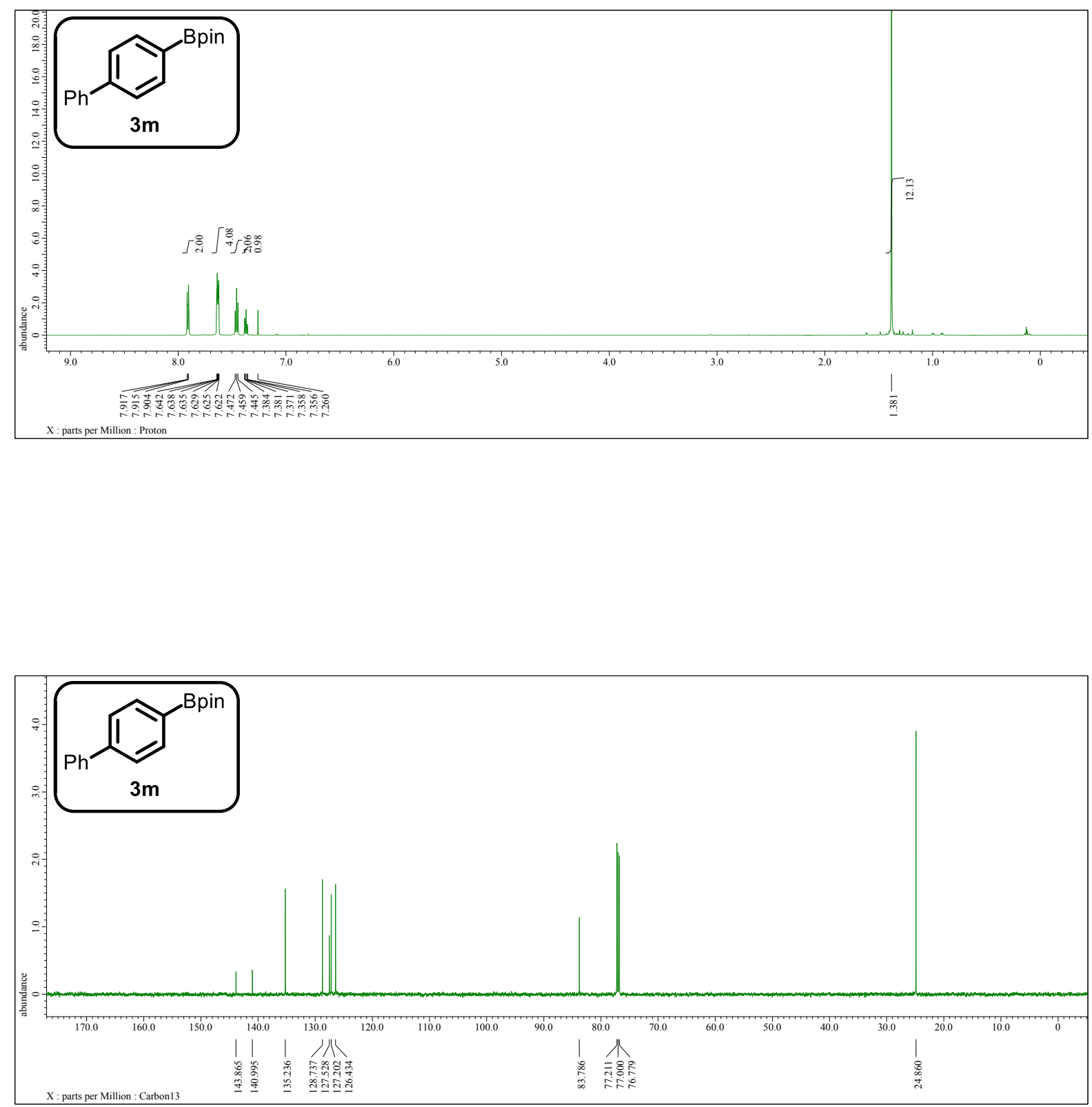

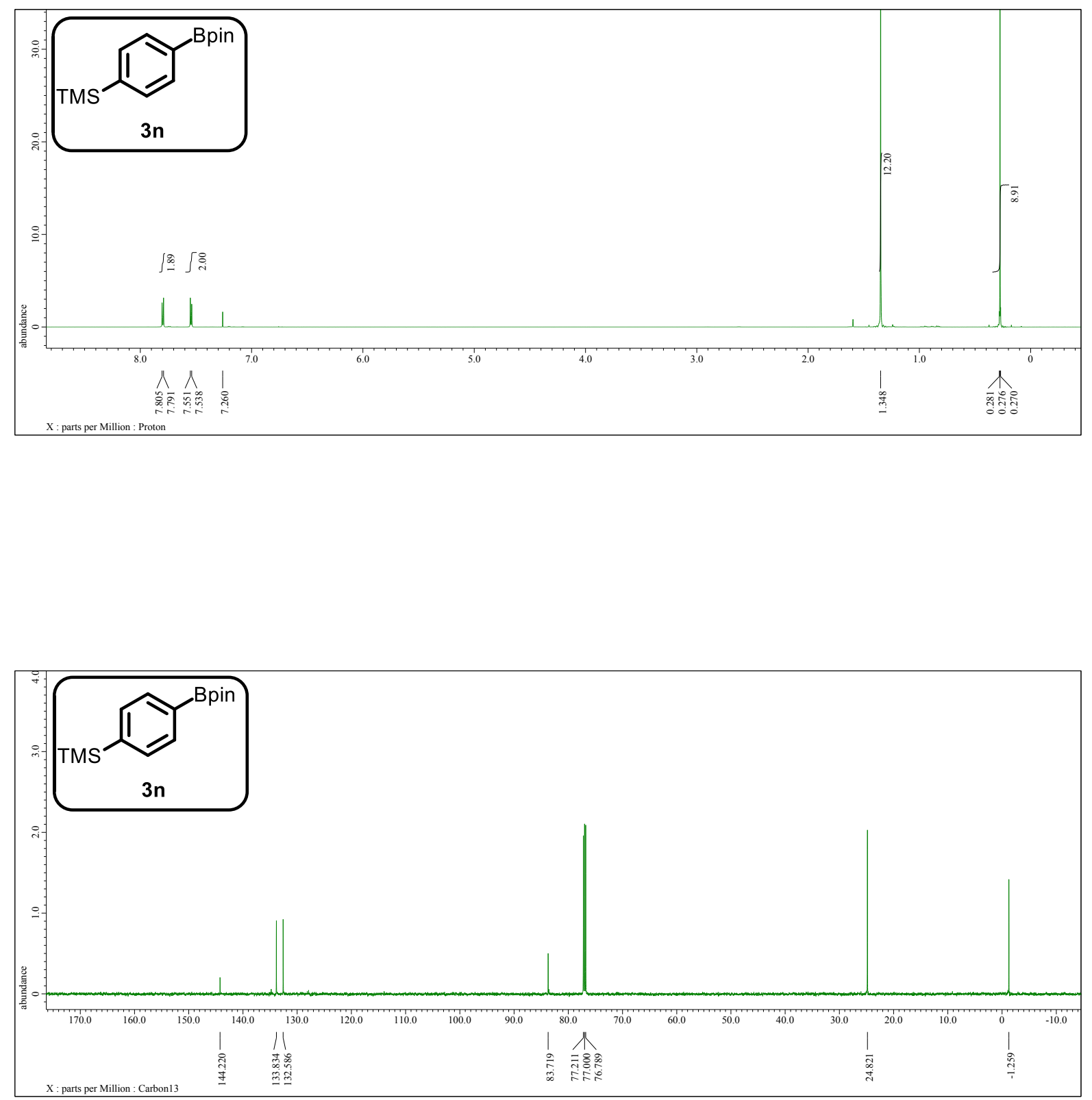

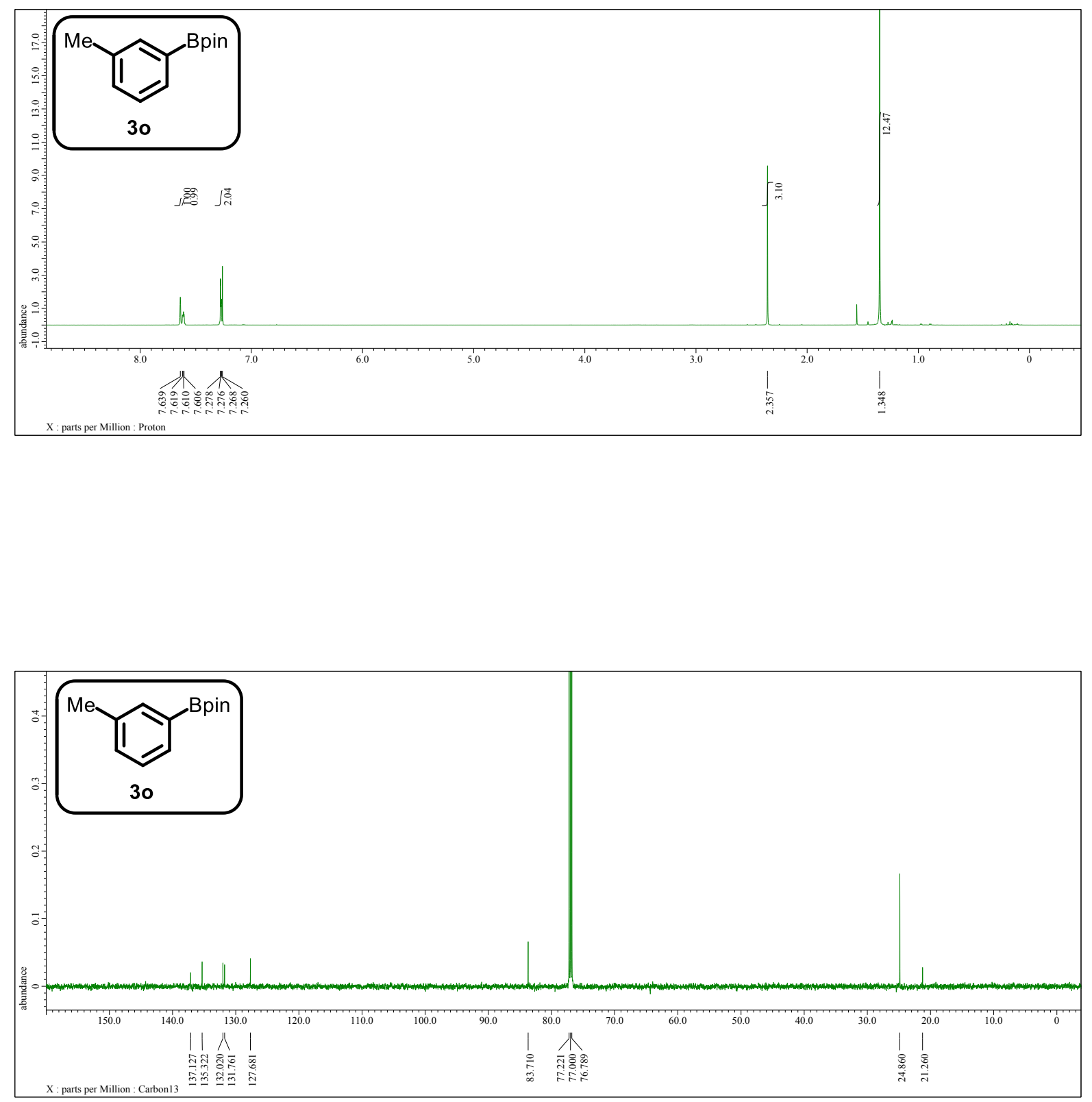

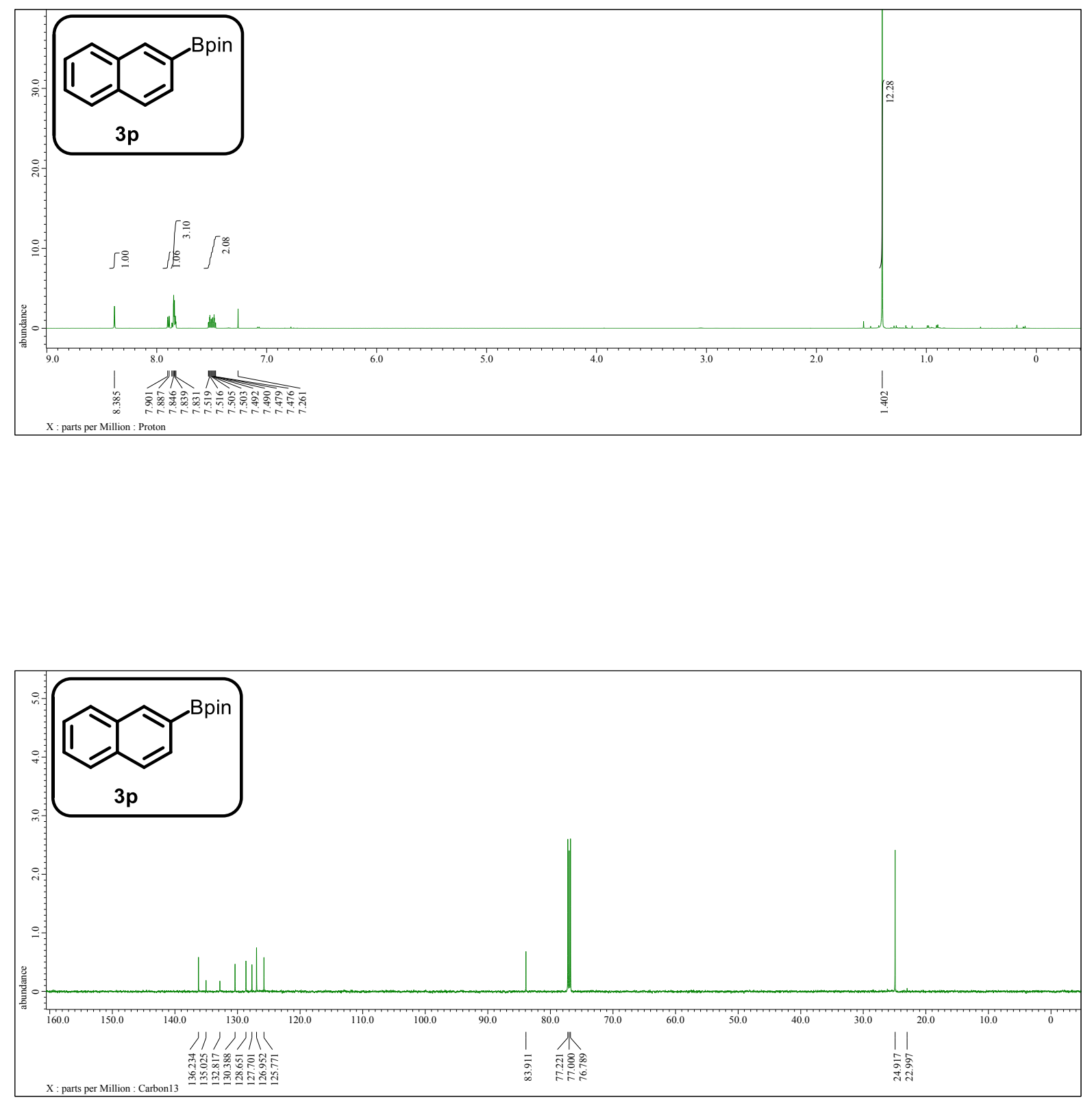

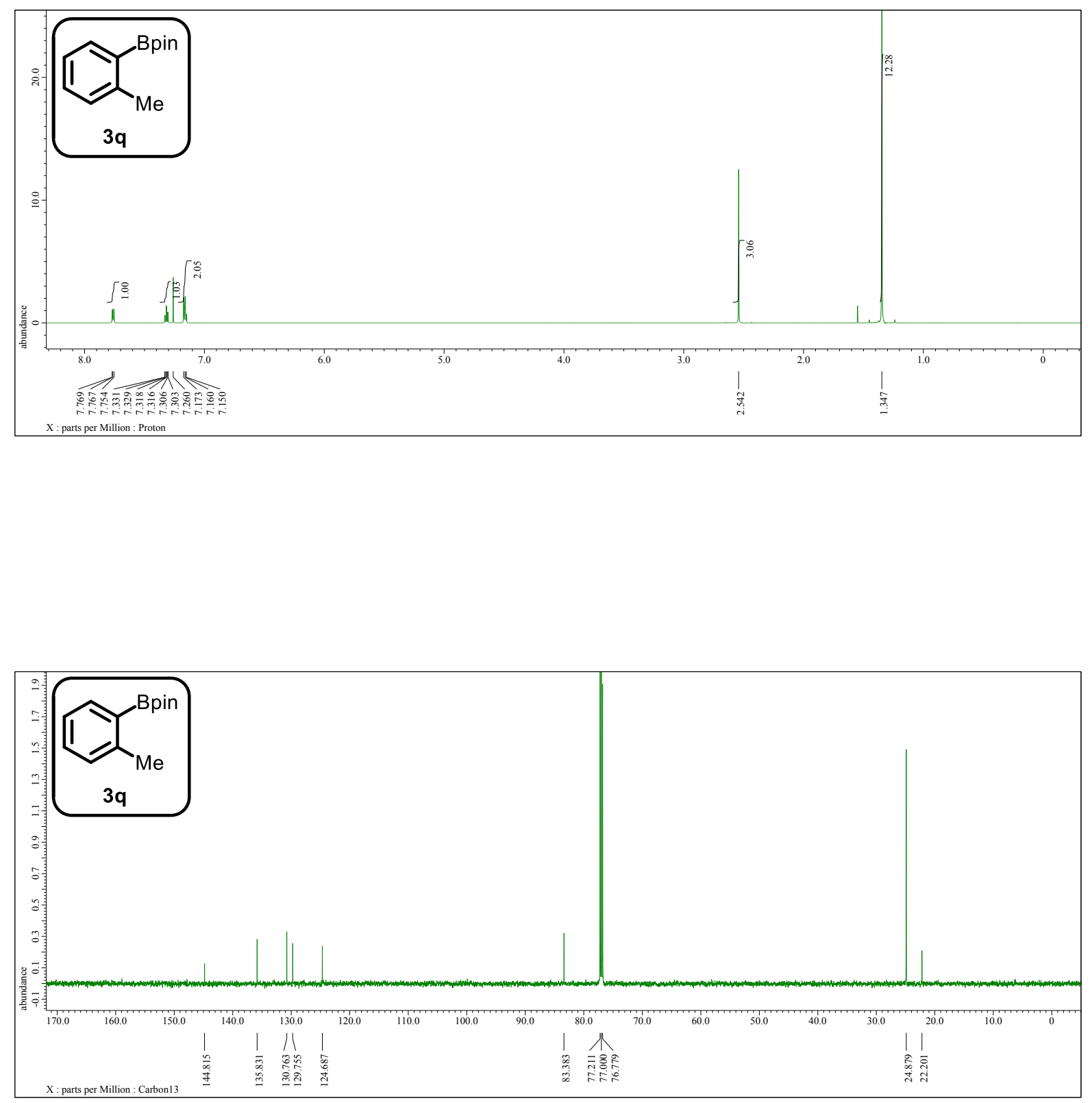

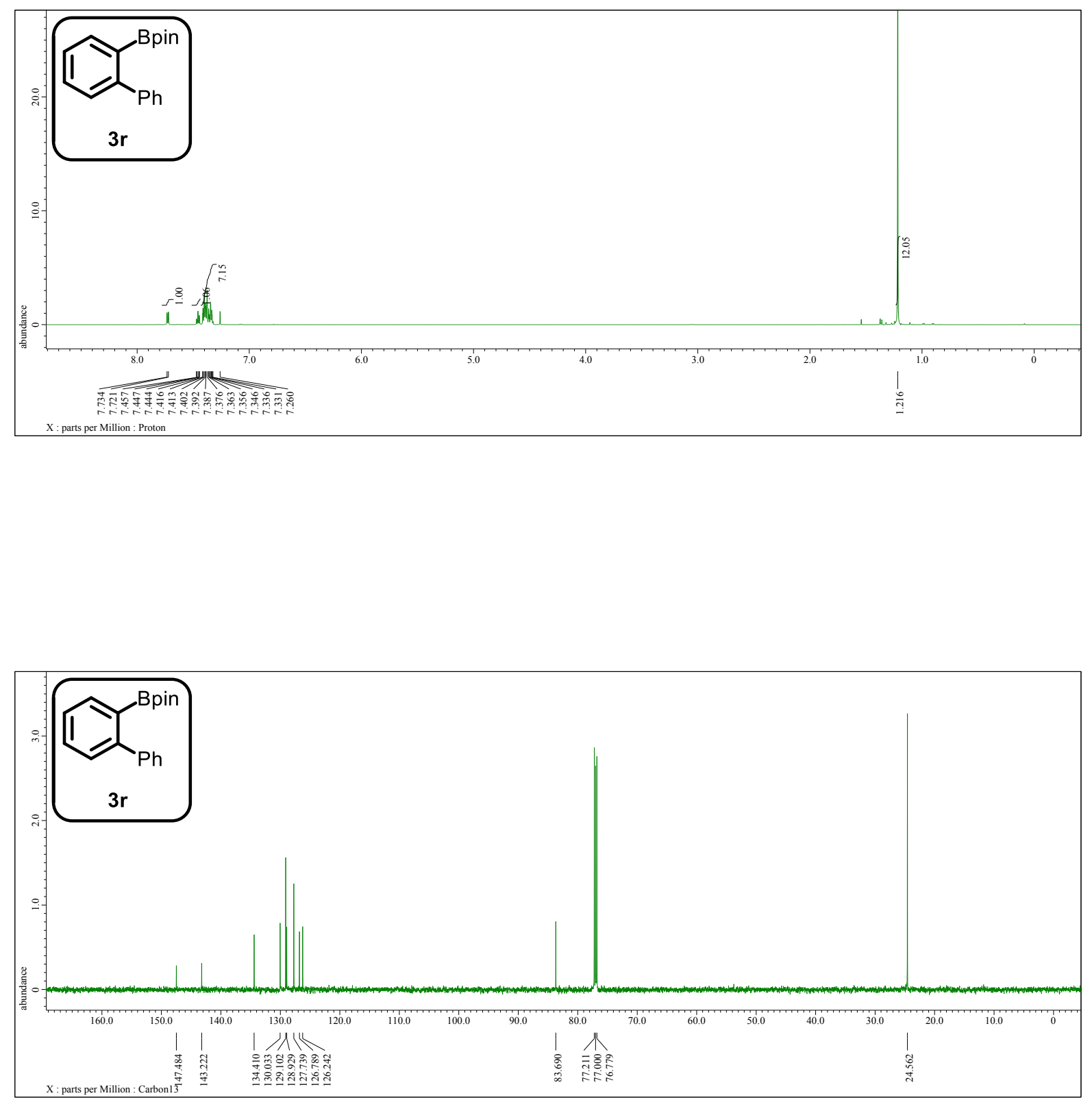

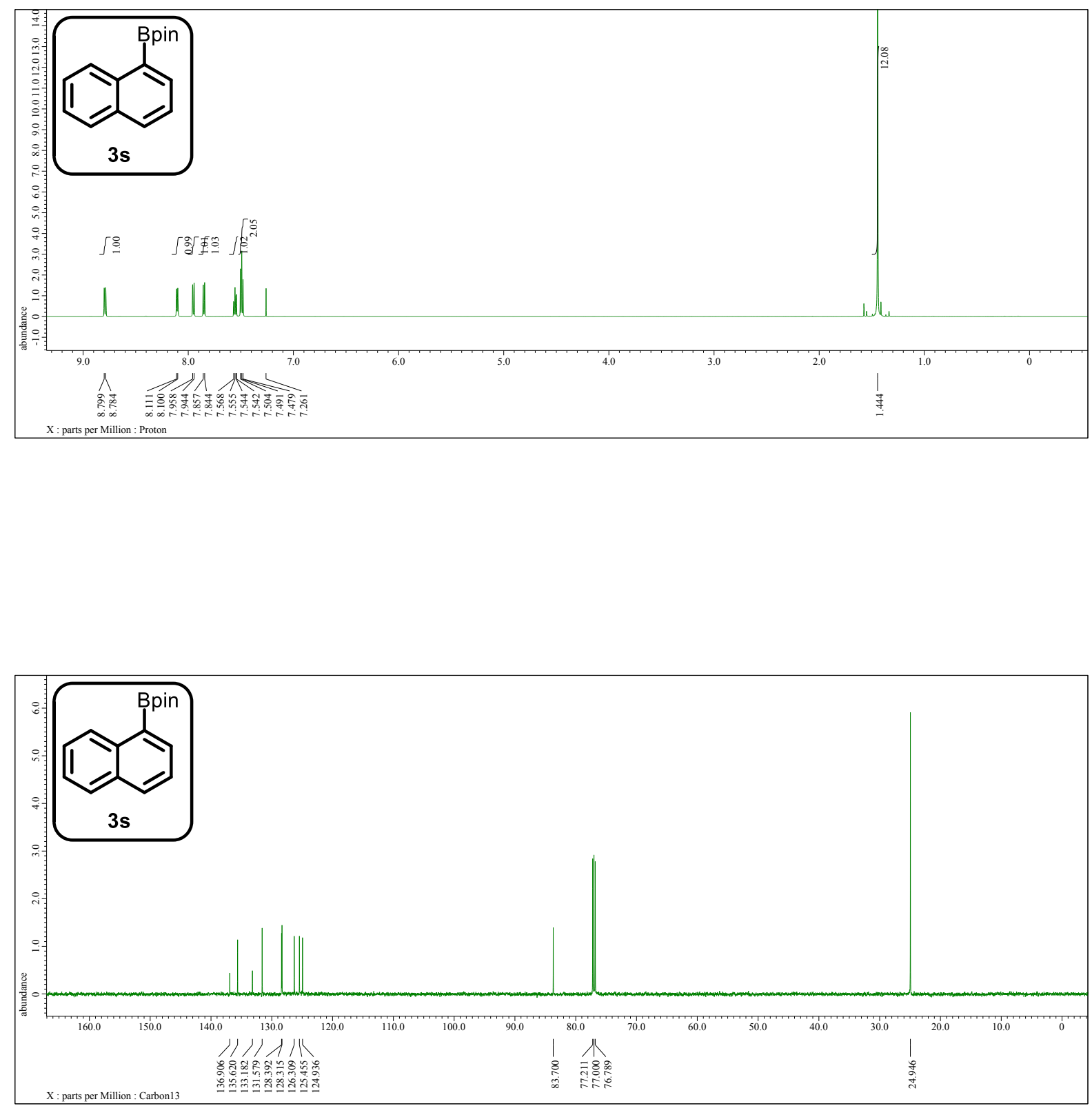

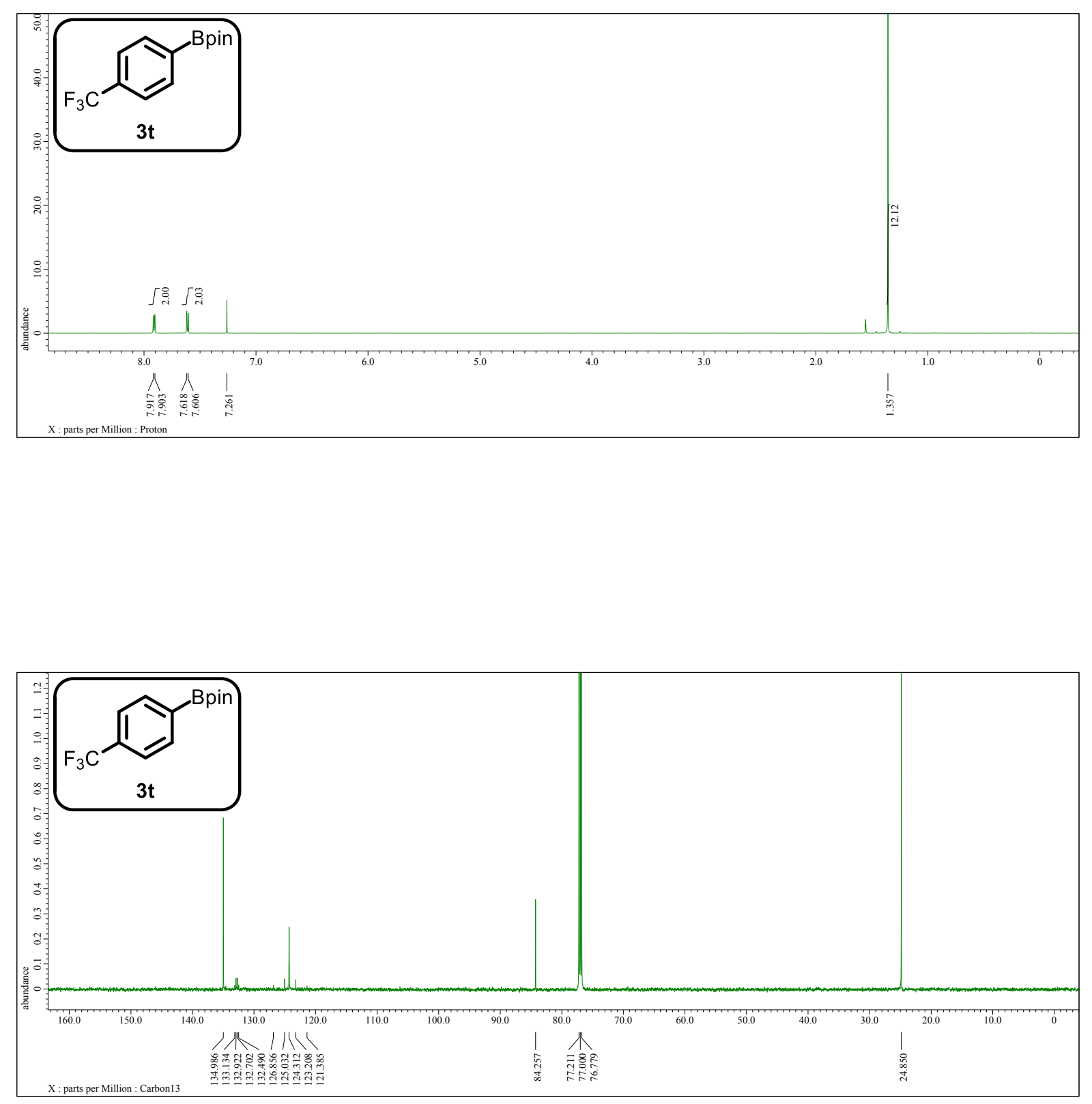

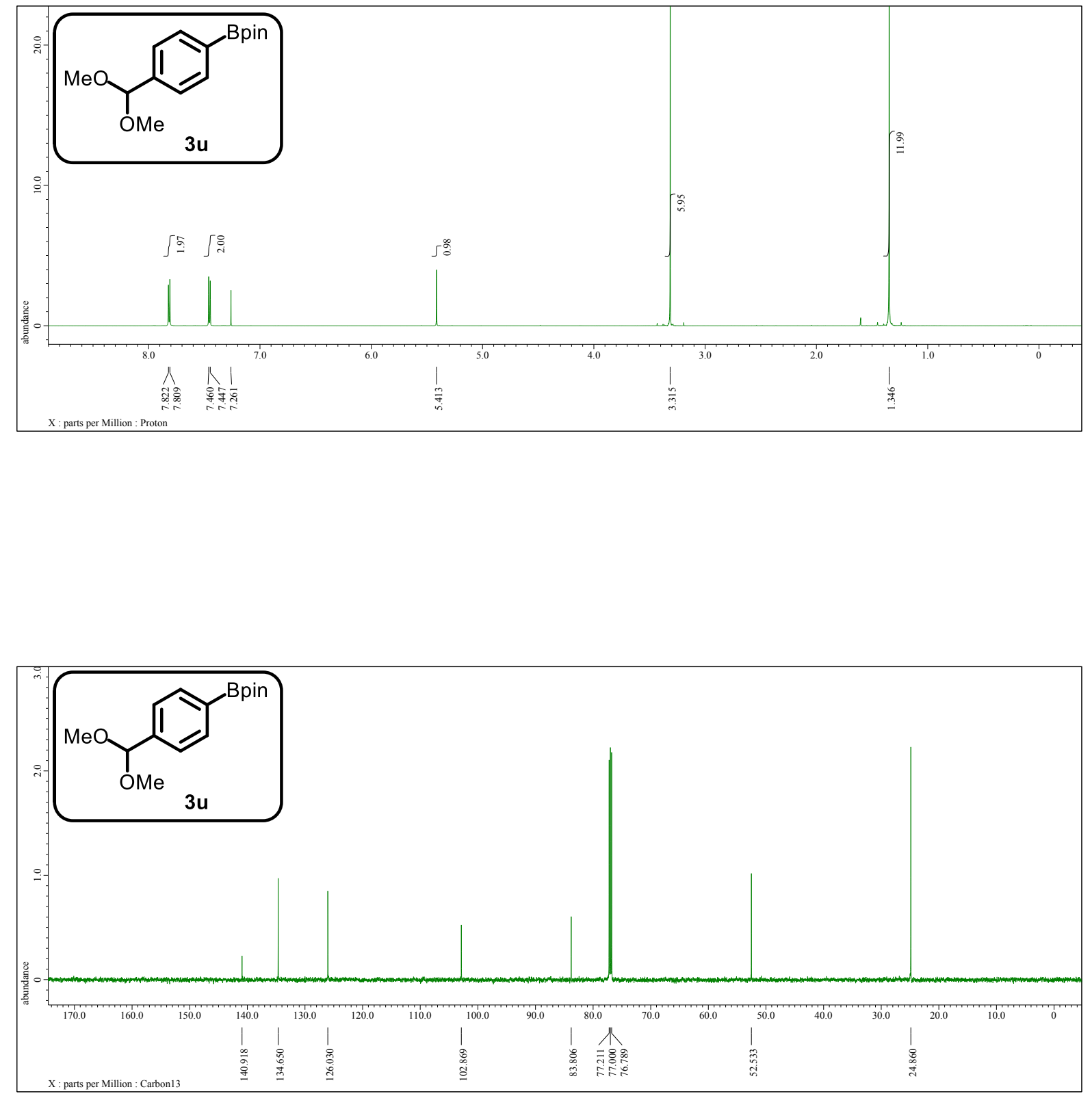

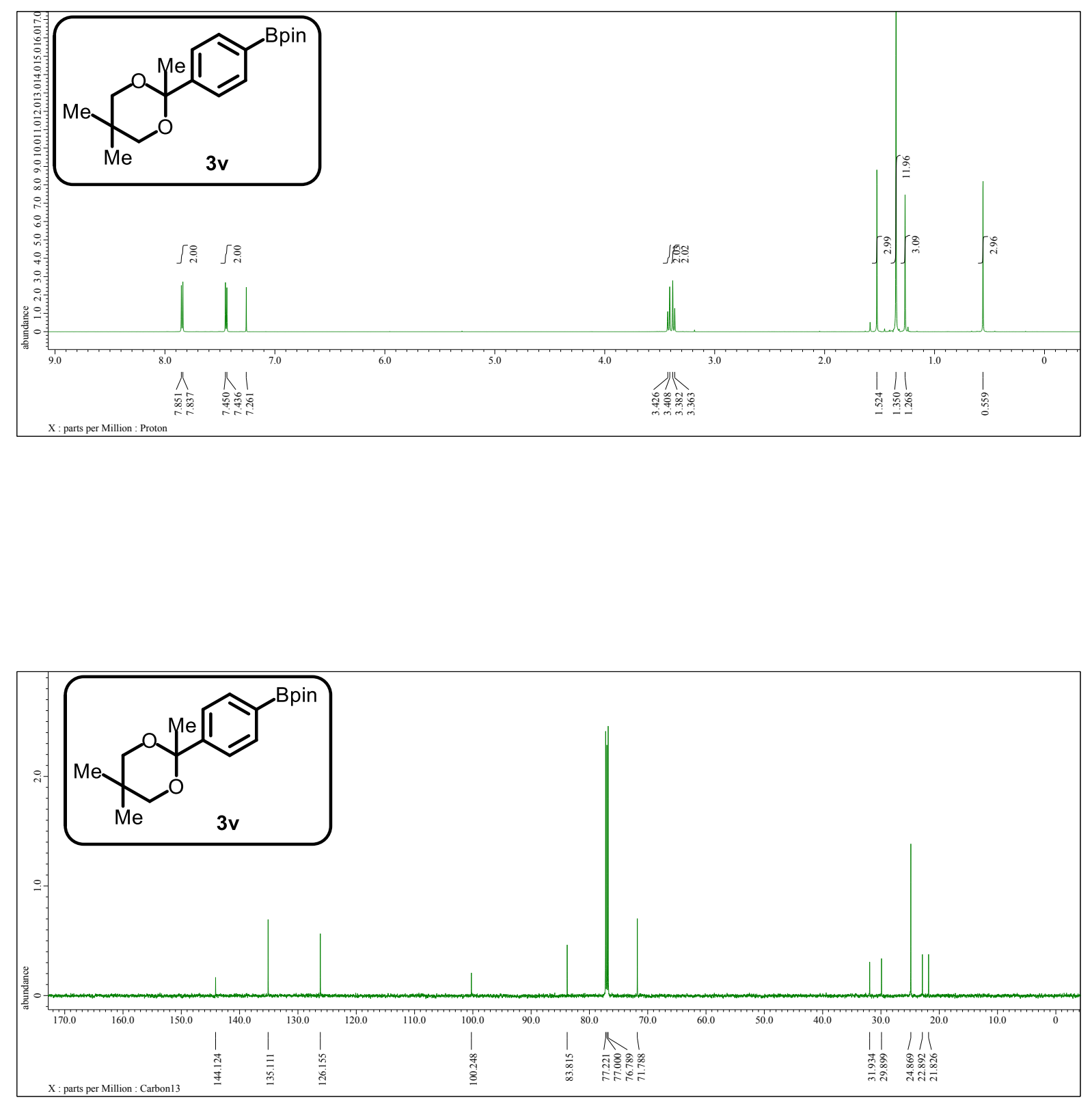

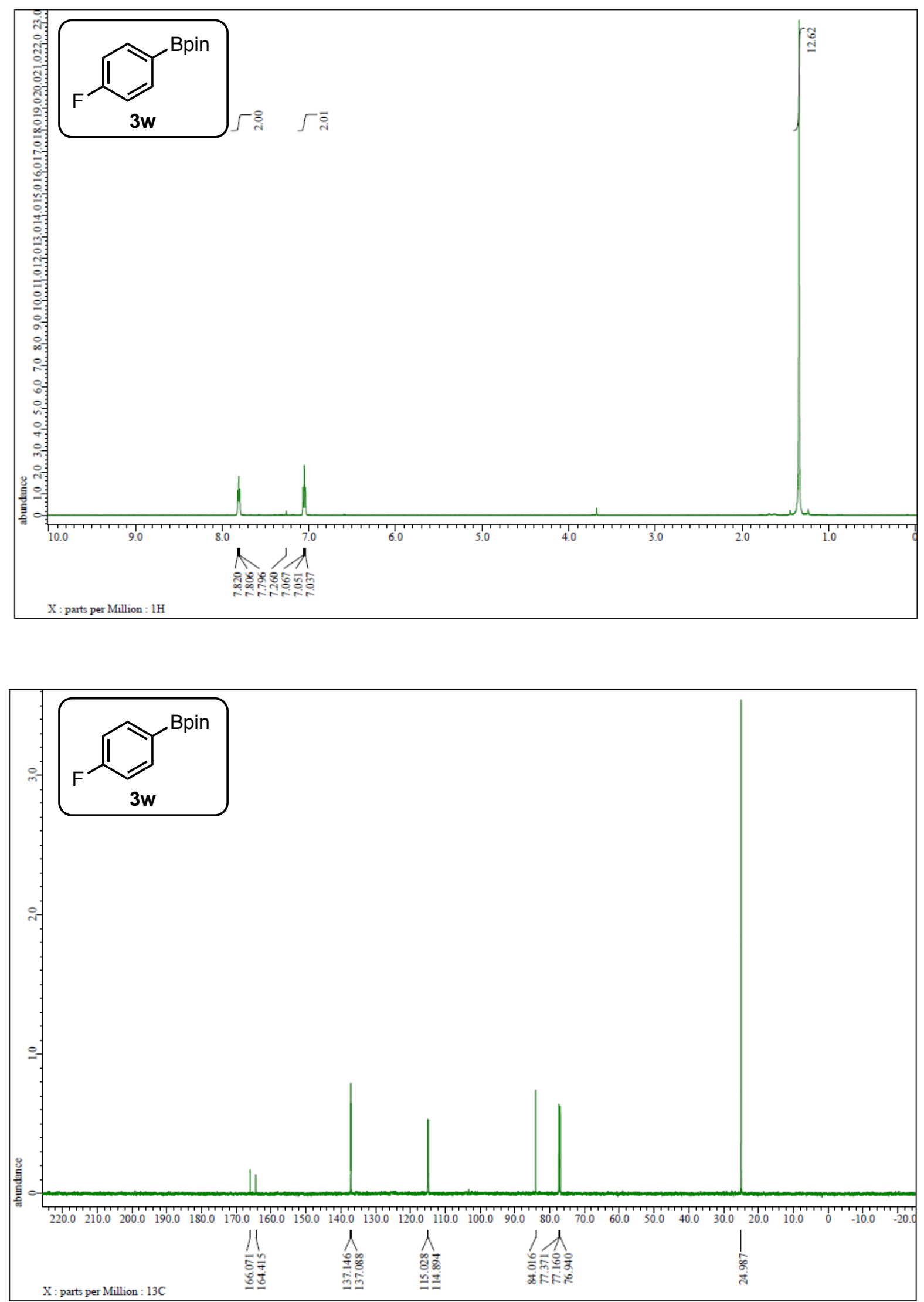

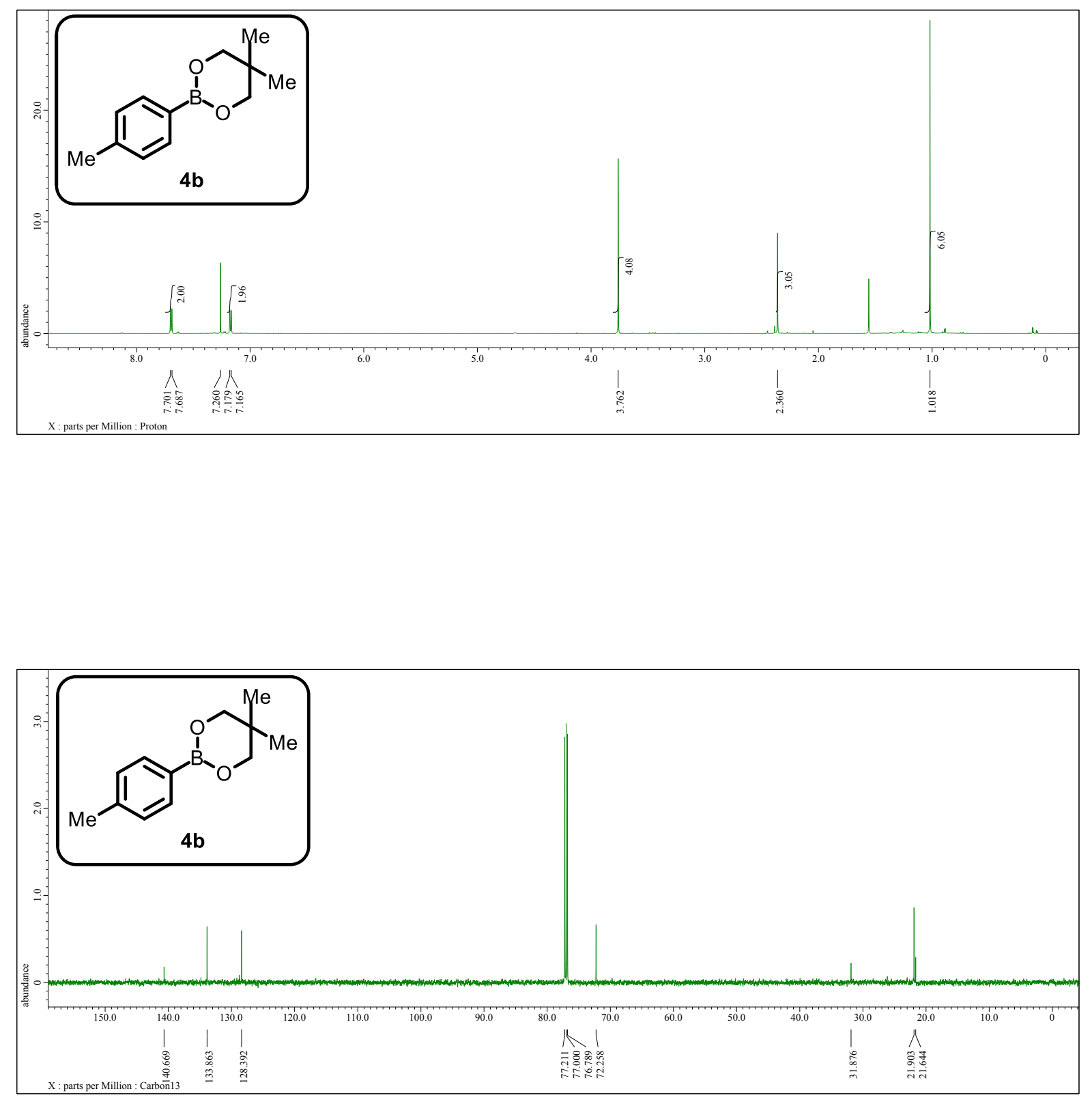

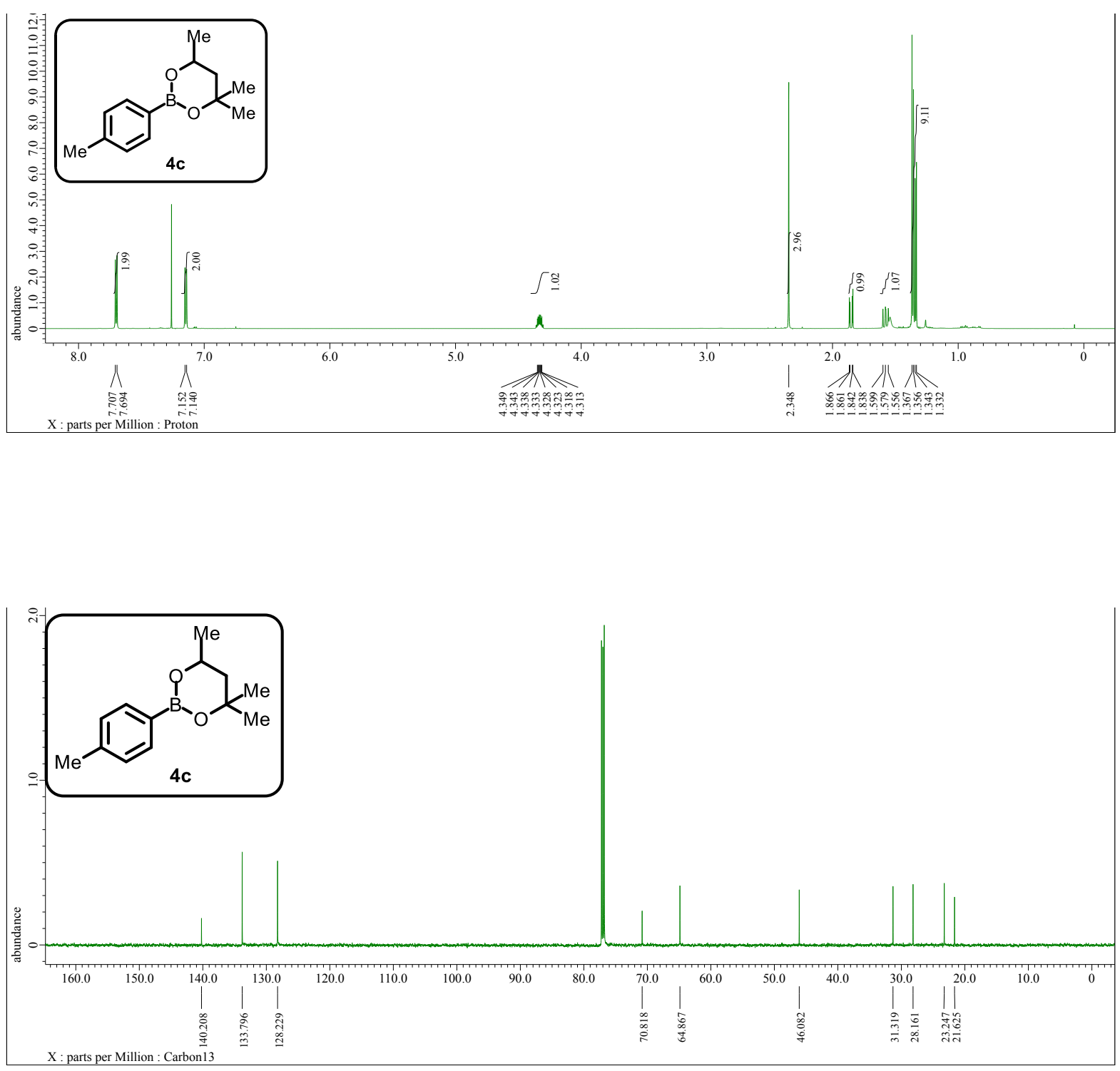